\title{
RESEARCH
}

\section{Goursat rigid local systems of rank four}

\author{
Danylo Radchenko ${ }^{1 *}$ (D) and Fernando Rodriguez Villegas ${ }^{2}$
}

*Correspondence:
danradchenko@gmail.com
${ }^{1}$ Max Planck Institute for
Mathematics, Vivatsgasse 7,
53111 Bonn, Germany
Dedicated to Don Zagier for his
65 th birthday
Full list of author information is
available at the end of the article

available at the end of the article

\begin{abstract}
We study the general properties of certain rank 4 rigid local systems considered by Goursat. We analyze when they are irreducible, give an explicit integral description as well as the invariant Hermitian form $H$ when it exists. By a computer search, we find what we expect are all irreducible such systems all whose solutions are algebraic functions and give several explicit examples defined over $\mathbb{Q}$. We also exhibit one example with infinite monodromy as arising from a family of genus two curves.
\end{abstract}

\section{Introduction}

The question of when linear differential equations in a variable $t$ have all of their solutions algebraic functions of $t$ goes back to the early 1800s. In his 1897 thesis, written under the supervision of P. Painlevé, Boulanger [4] mentions a paper by J. Liouville of 1833 [17] as a possible first work on the matter. The introduction of Boulanger's thesis offers a lucid description of the history of the question up to the time of his writing. For more recent work on the problem, see [26].

Schwarz [21] famously described all cases of algebraic solutions to the hypergeometric equation satisfied by Gauss's series ${ }_{2} F_{1}$. This was much later extended to hypergeometric equations of all orders by Beukers and Heckman [2]. In what follows, we will often refer to the better known hypergeometric local systems for comparison with [2] as our main source. For general background on local systems, monodromy representations and differential equations, see [6].

From a broader point of view, we may say that differential equations with all solutions algebraic are a special case of motivic local systems. Without attempting a rigorous definition of what this means, we will just say that such systems should be geometric in nature. Simpson conjectures in [25, p. 9] that all rigid local systems (see Sect. 2) satisfying some natural conditions are motivic. This is known for rigid local systems on $\mathbb{P}^{1}$ by the work of Katz [16], who gave a general algorithm (using middle convolution) for their construction. See also [8] for systems over a higher dimensional base and [29] for more on differential equations and arithmetic.

Goursat in his remarkable 1886 paper [10] discusses differential equations which, in later terminology, have no accessory parameters; i.e., where the local data uniquely determines the global monodromy representation. In this note, we consider his case II of rank 4 (denoted henceforth by G-II). These are order four linear differential equations in a variable $t$ with three regular singular points and semisimple local monodromies with

(c) The Author(s) 2018. This article is distributed under the terms of the Creative Commons Attribution 4.0 International License (http://creativecommons.org/licenses/by/4.0/), which permits unrestricted use, distribution, and reproduction in any medium, provided you give appropriate credit to the original author(s) and the source, provide a link to the Creative Commons license, and indicate if changes were made. 
eigenvalues of multiplicities $21^{2}, 2^{2}, 1^{4}$, respectively (see Sect. 7). We will fix the singular points to be $0,1, \infty$. In modern language, G-II corresponds to certain rigid local systems of rank 4. We will always assume the local monodromies are of finite order. This a necessary condition for a local system to be motivic [19, Thm. 9], the main focus of this paper. (Note that since we assume the local monodromies are semisimple finite order is equivalent to quasi-unipotent.)

In this paper, we study the general properties of G-II systems; for example, we analyze when they are irreducible and describe a Hermitian form $H$ invariant under the monodromy group when it exists. This is done in Sect. 4. As in [2], $H$ is a key tool to understand when this group is finite. Indeed, a necessary condition is that $H$ be definite in every complex embedding of the field of definition. Finiteness of the monodromy group is equivalent to solutions to the linear differential equations being algebraic.

We also show explicitly in Sect. 4.3 that the monodromy group can be defined in an integral way in terms of the eigenvalues of the local monodromies (the defining data). This abstractly follows from the fact that rigid systems over $\mathbb{P}^{1}$ are motivic (see [25, p. 9]); on the other hand, our construction is explicit. We find (see Sect. 3) that there is a nontrivial obstruction for the field of definition of the monodromy group. It may not be possible to define the monodromy group in its field of moduli (the field of coefficients of the characteristic polynomials of the local monodromies). This is in contrast with the hypergeometric case, for example, where by a theorem of Levelt [2, Prop. 3.3] such an obstruction does not occur. For the G-II systems, the obstruction is given by a quaternion algebra over the field of moduli (see the end of Sect. 4.3 for the general case and Sect. 8 for the case where the field of moduli is $\mathbb{Q}$ ).

As in the hypergeometric case, there are infinitely many cases of finite monodromy G-II local systems which come in families. These families depend linearly on a rational parameter. For G-II, there are two such families (see Sect. 12). All of these cases have imprimitive monodromy groups.

By a computer search, we find in Sect. 5 what we expect are all irreducible G-II equations whose solutions are algebraic functions and give several explicit examples defined over $\mathbb{Q}$ in Sect. 9. In Sect. 6, we show how some G-II cases can be constructed starting from a rank 4 Coxeter group by appropriate choices of pairs of commuting reflections. We exhibit in Sect. 11 one example with infinite monodromy as arising from a family of genus two curves.

We should point out that G-II is a special case of rigid local systems with at least one regular semisimple local monodromy. These were classified by Simpson in [24]. Except for a sporadic case in rank 6 they consist of the hypergeometric cases and one other case in each rank $\geq 2$. An explicit construction of the corresponding differential equations for these was given by [9]; see also [12] and [13].

We present in this paper our results with few detailed proofs, which will appear in a subsequent work. We used MAGMA [3] and PARI-GP [27] for most of the calculations.

\section{Rigid local systems}

Following the setup and notation of [14], we consider the character variety $\mathcal{M}_{\mu}$ where $\mu$ is an ordered $k$-tuple of partitions of a positive integer $n$. This variety parametrizes representations of $\pi_{1}(\Sigma \backslash S, *)$ to $\mathrm{GL}_{n}(\mathbb{C})$ mapping a small oriented loop around $s \in S$ to a semisim- 
ple conjugacy class $C_{s}$ whose generic eigenvalues have multiplicities $\mu^{s}=\left(\mu_{1}^{s}, \mu_{2}^{s}, \ldots\right)$, a corresponding partition in $\mu$. Unless otherwise stated in what follows conjugation will always refer to conjugation by $\mathrm{GL}_{n}(\mathbb{C})$.

Here $\Sigma$ is a Riemann surface of genus $g$ and $S$ is a finite set of $k$ points. The eigenvalues are assumed generic in the sense of [14]. If non-empty, the variety $\mathcal{M}_{\mu}$ is equidimensional of dimension

$$
d_{\mu}:=(2 g-2+k) n^{2}-\sum_{s \in S} \sum_{i \geq 1}\left(\mu_{i}^{s}\right)^{2}+2 .
$$

In this paper, we will only consider the case where $g=0$ and in detail when $k=3, n=4$ and, taking $S=\{0,1, \infty\}$, the partitions are $\mu^{0}=21^{2}, \mu^{1}=2^{2}, \mu^{\infty}=1^{4}$.

To be concrete, if $g=0$, given conjugacy classes $C_{1}, \ldots, C_{k} \subseteq \mathrm{GL}_{n}(\mathbb{C})$ and labeling the punctures with $1, \ldots, k$, we are looking for solutions to

$$
T_{1} \cdots T_{k}=I_{n}, \quad T_{s} \in C_{s}, \quad s=1, \ldots, k
$$

where $I_{n}$ is the identity matrix, up to simultaneous conjugation. Given such a representation $\pi_{1}(\Sigma \backslash S, *)$, we call the image in $\Gamma:=\left\langle T_{1}, \ldots, T_{k}\right\rangle \leq \mathrm{GL}_{n}(\mathbb{C})$ the (geometric) monodromy group. It is well defined up to conjugation.

Goursat in his remarkable 1886 paper [10] discusses when the local monodromy data uniquely determines the representation, or in terms of the differential equation and in later terminology, when are there no accessory parameters. We want local conditions that guarantee the following. Given two $k$-tuples of matrices $T_{s} \in C_{s}$ and $T_{s}^{\prime} \in C_{s}$ for $s \in S$ satisfying (1), there exists a single $U \in \mathrm{GL}_{n}(\mathbb{C})$ such that $T_{s}^{\prime}=U T_{s} U^{-1}$ for all $s \in S$. The corresponding local systems (determined by the local solutions to the linear differential equation) are known as rigid local systems [16].

To have a rigid local system is to say that $\mathcal{M}_{\mu}$ consists of a single point. Therefore, it is necessary that the expected dimension $d_{\mu}$ be zero. This is precisely Goursat's condition $[10,(5)$ p.113] (he only considers the case of $g=0$ ) as well as Katz's [16], which follows from cohomological considerations.

We assume from now on that $g=0$ and then to avoid trivial cases that $k \geq 3$. Indeed, for $g=0, k=1$, the group $\pi_{1}(\Sigma \backslash S, *)$ is trivial and for $g=0, k=2$ it is isomorphic to $\mathbb{Z}$. Note, as Goursat points out, that adding an extra puncture to $S$ with associated partition (n) does not change the value of $d_{\mu}$. Such points correspond to apparent singularities in the differential equation and may hence be safely ignored. We will assume then that the partitions $\mu_{s}$ have at least two parts.

Goursat shows that with the given assumptions $k \leq n+1$ [10, top p.114] and hence there are only finitely many solutions of $d_{\mu}=0$ for fixed $n$. He lists [10, p. 115] the cases of $d_{\mu}=0$ for $n=3$ and $n=4$ (see below).

It turns out, however, that the condition $d_{\mu}=0$ is not sufficient as the variety $\mathcal{M}_{\mu}$ might be empty. Crawley-Boevey [5] proved that a necessary and sufficient condition for $\mathcal{M}_{\mu}$ to be a point, in the case of generic eigenvalues we are considering, is that $\mu$ corresponds to a real root of the associated Kac-Moody algebra. Without getting too deeply into the details of this condition, we present an algorithm that will allow us to determine when $\mathcal{M}_{\mu}$ is a point. This algorithm ultimately corresponds to Katz's middle convolution and is simply an explicit implementation of Crawley-Boevey's criterion. The reader may consult [18] as a general reference for this topic. 
It is more convenient to present the multiplicity data $\mu$ in the form of a star graph with one central node and $k$ legs (see [5] and also [14]). We illustrate this in our basic case G-II (Goursat's label II for $n=4$ ).

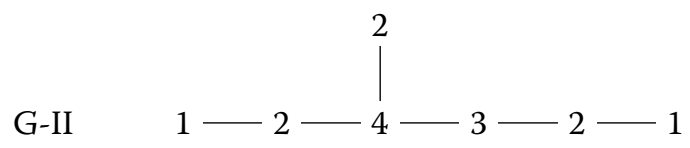

The partitions can be read by starting at the central node and moving away along a leg. The successive differences of the respective node values are the parts of the corresponding partition. Nodes with a zero value are not included. For example, in the diagram for G-II given above, there are three legs. The leg to the left has nodes 4, 2, 1 corresponding to the partition $(2,1,1)$ since $4-2=2,2-1=1,1-0=1,0-0=0, \ldots$. Similarly, the vertical leg represents the partition $(2,2)$ and the leg to the right $(1,1,1,1)$.

The algorithm proceeds starting from a configuration as above corresponding to an ordered $k$-tuple $\mu$ of partitions of $n$ satisfying $d_{\mu}=0$ using the following moves.

- A: Replace the value $n$ at the central node by

$$
\sum_{i} n_{i}-n
$$

where $n_{i}$ are the values at the nodes closest to the central node.

- B: Shrink to a point any segment whose endpoints values are the same.

- C: For each leg put new values on the nodes (not including the central node) so that the set of differences of consecutive values remains the same but appear in nondecreasing order as one moves away from the central node along the leg (so that they correspond to a partition of the value at the central node).

The goal is to use a sequence of these moves to reach the terminal configuration of just a central node with value 1 . Under the assumptions $d_{\mu}=0, g=0$ applying A strictly decreases the value at the central node and hence the algorithm always terminates. Indeed, for any partition $\mu=\mu_{1} \geq \mu_{2} \geq \cdots$ of $n$, we have $n \mu_{1} \geq \sum_{i} \mu_{i}^{2}$. It follows that if $d_{\mu}=0$

$$
n \sum_{s} \mu_{1}^{s}>(2 g-2+k) n^{2}
$$

and since also $g=0$ and $n>0$ that $\sum_{s} \mu_{1}^{s}>(k-2) n$ which proves the claim.

For our running example $\mu=\left(21^{2}, 2^{2}, 1^{4}\right)$, the algorithm works as follows.

Apply A:

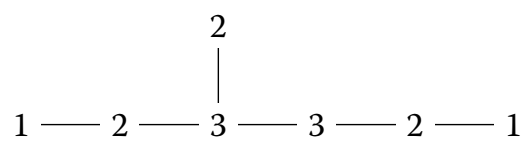

Apply B:

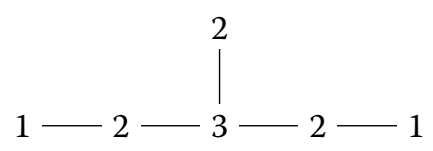


Apply C:

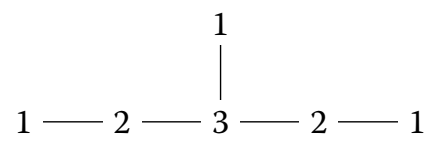

We have arrived at the case $\mu=\left(1^{3}, 21,1^{3}\right)$ that corresponds to the hypergeometric equation of order 3. It is easy to see that a next stage takes us to $\mu=\left(1^{2}, 1^{2}, 1^{2}\right)$ corresponding to the hypergeometric equation of rank 2 and finally to the desired terminal case. This confirms that indeed G-II corresponds to a rigid local system.

The algorithm fails if at any stage we cannot perform C; i.e., applying A yields a graph with a central value strictly smaller than one of its neighbors. This indeed happens for Goursat's case IV as we verify below.

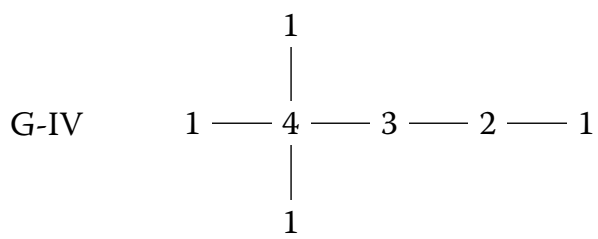

Apply A:

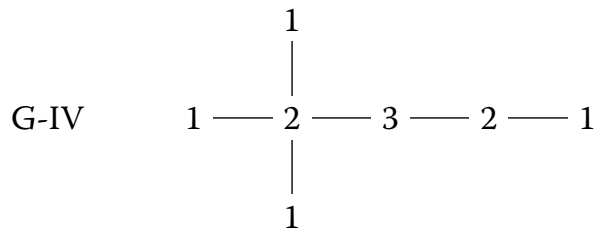

Since $2<3$, we cannot apply $\mathrm{C}$ on the leg going off to the right. (One of the parts would have to be $2-3=-1$.)

We should note that Goursat himself showed using classical tools that his case IV did not correspond to a differential equation without accessory parameters [10, p. 120] (...on devra exclure la quatrième).

Here are the diagrams of all rank $n=4$ rigid local systems of the type in question and their corresponding label in Goursat's paper (all but the case IV just discussed actually correspond to a rigid local system).

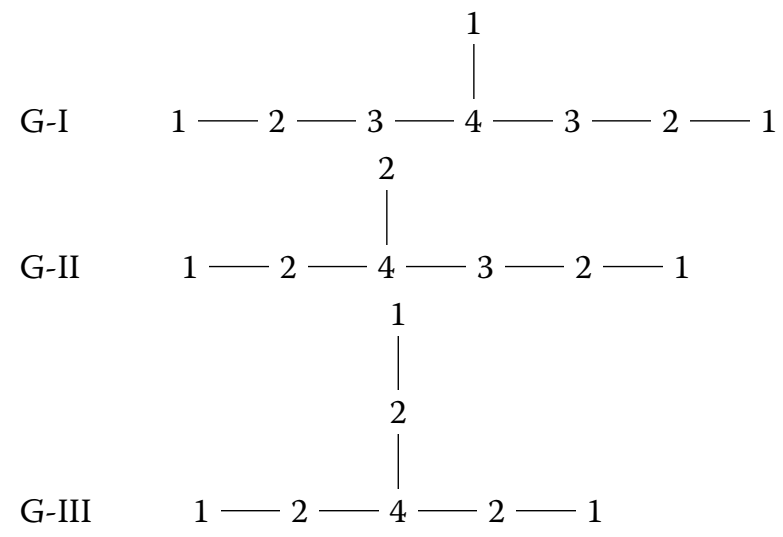




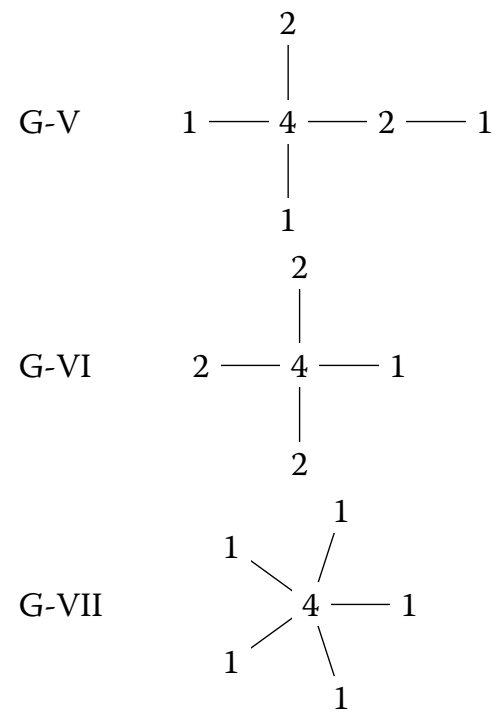

\section{Field of definition and field of moduli}

Given a rigid local system with conjugacy classes $C_{s}$ for $s \in S$ as in Sect. 2, let $q_{s}(T)$ be the characteristic polynomial of any element of $C_{s}$. Let $K$ be the field obtained by adjoining to $\mathbb{Q}$ the coefficients of all $q_{s}$. We call $K$ the field of moduli or simply the trace field of the local system (see below for a justification for this name). It is the smallest field $F$ over which local monodromies $T_{s} \in \mathrm{GL}_{n}(F)$ of the required kind, i.e., $T_{s} \in C_{s}$, may exist. But as is typical in such problems it does not mean that we can actually choose $F=K$.

Given a collection of local monodromies giving rise to our local system, we call its field of definition the smallest extension $F$ of $\mathbb{Q}$ containing all of their entries. We necessarily have $K \subseteq F$. Note that by Levelt's theorem [2, Prop. 3.3], in the hypergeometric case, we can always take $F=K$, but this is not the case for Goursat's case II that we analyze here (see Sect. 4.3).

Let $T_{s} \in C_{s}$ be a $k$-tuple of matrices in $\mathrm{GL}_{n}(\overline{\mathbb{Q}})$ satisfying (1). It is clear that for $\sigma \in$ $\operatorname{Gal}(\bar{Q} / K)$ the $k$-tuple $T_{s}^{\sigma}$ is another solution to (1). Hence by rigidity, there exists $X_{\sigma} \in$ $\mathrm{GL}_{n}(\overline{\mathbb{Q}})$ such that

$$
X_{\sigma}^{-1} T_{s} X_{\sigma}=T_{s}^{\sigma}, \quad s \in S
$$

Again by rigidity, we find that there exists $a_{\sigma, \tau} \in \overline{\mathbb{Q}}$ such that

$$
X_{\sigma} X_{\tau}^{\sigma}=a_{\sigma, \tau} X_{\sigma \tau} .
$$

The map $(\sigma, \tau) \mapsto a_{\sigma, \tau}$ is a 2-cocycle giving a well-defined element $\xi \in H^{2}\left(\operatorname{Gal}(\bar{K} / K), K^{\times}\right)$.

The following is a consequence of a standard result in Galois cohomology (see [23, Chap.10, \$5]); we leave the details to the reader.

Proposition 1 There exists a solution to (1) over $K$ if and only if $\xi$ is trivial.

Note that (2) implies that the trace of any product of $T_{s}$ 's is in the trace field $K$. That is, $K$ is indeed the smallest extension of $\mathbb{Q}$ containing the traces of all $T \in \Gamma$. 


\section{Explicit solution for the Goursat case II}

In [10, p. 131], Goursat writes down an explicit solution to (1) for $S=\{0,1, \infty\}$ in the case when $T_{0}, T_{1}$, and $T_{\infty}$ are diagonalizable with spectra $1^{2} a_{1} a_{2}, 1^{2} b^{2}$ and $c_{1} c_{2} c_{3} c_{4}$, respectively (assuming that eigenvalues with different labels are distinct and that $a_{1} a_{2} b^{2} c_{1} c_{2} c_{3} c_{4}=1$ ). The characteristic polynomials $q_{s}$ of $T_{s}$ are therefore

$$
\begin{aligned}
q_{0}(T) & =(T-1)^{2}\left(T-a_{1}\right)\left(T-a_{2}\right), \\
q_{1}(T) & =(T-1)^{2}(T-b)^{2}, \\
q_{\infty}(T) & =\left(T-c_{1}\right)\left(T-c_{2}\right)\left(T-c_{3}\right)\left(T-c_{4}\right) .
\end{aligned}
$$

Sometimes it is more convenient to work with the characteristic exponents instead of the eigenvalues. We will use Greek letters to denote them, so that $a_{j}=\exp \left(2 \pi i \alpha_{j}\right)$ and similarly for $\beta$ 's and $\gamma$ 's.

Since the triple $\left(T_{0}, T_{1}, T_{\infty}\right)$ is irreducible, the 1-eigenspaces for $T_{0}$ and $T_{1}$ must have a zero intersection. Goursat then shows that in a suitable basis the matrices $T_{0}$ and $T_{1}$ are given by

$$
T_{0}=\left(\begin{array}{cccc}
1 & 0 & A\left(1-a_{1}\right) & B\left(1-a_{2}\right) \\
0 & 1 & C\left(1-a_{1}\right) & D\left(1-a_{2}\right) \\
0 & 0 & a_{1} & 0 \\
0 & 0 & 0 & a_{2}
\end{array}\right), \quad T_{1}=\left(\begin{array}{cccc}
b & 0 & 0 & 0 \\
0 & b & 0 & 0 \\
1-b & 0 & 1 & 0 \\
0 & 1-b & 0 & 1
\end{array}\right)
$$

A direct computation shows that for given $a_{1}, a_{2}$, and $b$, the coefficients of $q_{\infty}$ depend linearly on $A, D$, and $A D-B C$. Conversely, the numbers $A, D$, and $A D-B C$ can be found from $q_{\infty}$ by

$$
\begin{aligned}
& \frac{(b-1)\left(a_{1}-1\right)\left(a_{2}-a_{1}\right)}{b^{2} a_{1}^{2} a_{2}} A=\frac{a_{1}^{2} q_{\infty}\left(a_{1}^{-1}\right)-b^{2} q_{\infty}\left(b^{-1}\right)}{a_{1}-b}, \\
& \frac{(b-1)\left(a_{2}-1\right)\left(a_{1}-a_{2}\right)}{b^{2} a_{1} a_{2}^{2}} D=\frac{a_{2}^{2} q_{\infty}\left(a_{2}^{-1}\right)-b^{2} q_{\infty}\left(b^{-1}\right)}{a_{2}-b}, \\
& \frac{(b-1)^{2}\left(a_{1}-1\right)\left(a_{2}-1\right)}{b^{2} a_{1} a_{2}}(A D-B C)=b^{2} q_{\infty}\left(b^{-1}\right) .
\end{aligned}
$$

In particular, these identities imply that $A, D$, and $B C$ are uniquely determined from the spectra. On the other hand, conjugation by the diagonal matrix $D=\operatorname{diag}(\lambda, 1, \lambda, 1)$ preserves the shapes of $T_{0}$ and $T_{1}$ and maps $(B, C)$ to $\left(\lambda^{-1} B, \lambda C\right)$, hence only the product $B C$ is uniquely determined.

\subsection{Criterion for irreducibility}

We now find a criterion for when the constructed representation is irreducible. The eigenmatrices for $T_{0}$ and $T_{1}$ are

$$
Z_{0}=\left(\begin{array}{cccc}
1 & 0 & -A & -B \\
0 & 1 & -C & -D \\
0 & 0 & 1 & 0 \\
0 & 0 & 0 & 1
\end{array}\right), \quad Z_{1}=\left(\begin{array}{cccc}
0 & 0 & -1 & 0 \\
0 & 0 & 0 & -1 \\
1 & 0 & 1 & 0 \\
0 & 1 & 0 & 1
\end{array}\right)
$$


One can easily check the following assertions: if $C=0$, then the subspace of vectors of the form $(*, 0, *, 0)$ is invariant; if $B=0$, then the subspace $(0, *, 0, *)$ is invariant; if $A D-B C=0$, then the subspace spanned by $\operatorname{ker}\left(T_{1}-I\right)$ and the vector $(a, c, 0,0)$ is invariant; if $A D-B C-A-D+1=0$, then the subspace spanned by $\operatorname{ker}\left(T_{1}-b I\right)$ and the vector $(a-1, c, 0,0)$ is invariant. Conversely, if $V$ is a non-trivial invariant subspace, then considering the various possibilities for $V$ with respect to the eigenspaces of $T_{0}$, we find that one of $B, C, A D-B C$, or $A D-B C-A-D+1$ must vanish.

Thus, the representation is irreducible if and only if

$$
B C(A D-B C)(A D-B C-A-D+1) \neq 0 .
$$

To get the description in terms of eigenvalues, we use the following factorizations:

$$
\begin{aligned}
A D-B C & =\frac{a_{1} a_{2}\left(1-b c_{1}\right)\left(1-b c_{2}\right)\left(1-b c_{3}\right)\left(1-b c_{4}\right)}{(1-b)^{2}\left(1-a_{1}\right)\left(1-a_{2}\right)}, \\
B C & =\frac{b a_{2}^{3} \prod_{1 \leq i<j \leq 4}\left(1-a_{1} b c_{i} c_{j}\right)}{\left(a_{1}-a_{2}\right)^{2}(1-b)^{2}\left(1-a_{1}\right)\left(1-a_{2}\right)}, \\
A D-B C-A-D+1 & =\frac{\left(1-c_{1}\right)\left(1-c_{2}\right)\left(1-c_{3}\right)\left(1-c_{4}\right)}{c_{1} c_{2} c_{3} c_{4}(1-b)^{2}\left(1-a_{1}\right)\left(1-a_{2}\right)} .
\end{aligned}
$$

Note that in terms of $q_{\infty}$ this simply becomes $q_{\infty}(1)=0, q_{\infty}\left(b^{-1}\right)=0$, and $w_{2}\left(q_{\infty}\right)\left(a_{1}^{-1} b^{-1}\right)=0$, where $w_{2}\left(q_{\infty}\right)=\prod_{i<j}\left(T-c_{i} c_{j}\right)$ is the polynomial whose roots are products of all pairs of roots of $q_{\infty}$. This description agrees with the conditions given in $[20$, p. 10].

To summarize, let

$$
\mathbb{T}:=\left\{\left(a_{1}, a_{2}, b, c_{1}, \ldots, c_{4}\right) \mid a_{1} a_{2} b^{2} c_{1} \cdots c_{4}=1\right\} \subseteq S^{1} \times \cdots \times S^{1} \backslash \Delta
$$

be the space of eigenvalues (taken in the unit circle). Here $\Delta$ is the union of $a_{1}=$ $1, a_{2}=1, a_{1}=a_{2}, b=1$ and $c_{i}=c_{j}$ for $1 \leq i<j \leq 4$ guaranteeing that $\left(1,1, a_{1}, a_{2}\right),(1,1, b, b),\left(c_{1}, \ldots, c_{4}\right)$ are the eigenvalues of a G-II system. Define $\mathbb{T}^{\text {irr }}$ as the subset corresponding to irreducible local systems. Then we have

$$
\mathbb{T}^{\mathrm{irr}}=\mathbb{T} \backslash\left\{q_{\infty}(1) q_{\infty}\left(b^{-1}\right) w_{2}\left(q_{\infty}\right)\left(a_{1}^{-1} b^{-1}\right)=0\right\} .
$$

The conditions for irreducibility we found can also be obtained from [5, Thm. 1.5]. Indeed the required decompositions of the real root corresponding to G-II are the following (and their refinements). Let $i_{1}, \ldots, i_{4}$ be any re-ordering of $1, \ldots, 4$.

i) $q_{\infty}(1)=0$

$$
\begin{array}{ccc|cc}
1 & a_{1} & a_{2} & 1 \\
1 & b & b & 1, & a_{1} a_{2} b^{2} c_{i_{1}} c_{i_{2}} c_{i_{3}}=1, \quad c_{i_{4}}=1 . \\
c_{i_{1}} & c_{i_{2}} & c_{i_{3}} & c_{i_{4}}
\end{array}
$$

ii) $q_{\infty}\left(b^{-1}\right)=0$

\begin{tabular}{lll|l}
1 & $a_{1}$ & $a_{2}$ & 1
\end{tabular}

\begin{tabular}{ccc|cc|c}
1 & 1 & $b$ & $b$, & $a_{1} a_{2} b c_{i_{1}} c_{i_{2}} c_{i_{3}}=1, \quad b c_{i_{4}}=1$. \\
$c_{i_{1}}$ & $c_{i_{2}}$ & $c_{i_{3}}$ & $c_{i_{4}}$ & $a_{1}$
\end{tabular}

iii) $w_{2}\left(q_{\infty}\right)\left(a_{1}^{-1} b^{-1}\right)=0$

$$
\begin{array}{cc|cc}
1 & a_{1} & 1 & a_{2} \\
1 & b & 1 & b, \\
c_{i_{1}} & c_{i_{2}} & c_{i_{3}} & c_{i_{4}}
\end{array}
$$




\subsection{Invariant Hermitian form}

Let $T_{1}, \ldots, T_{k} \in \mathrm{GL}_{n}(\mathbb{C})$ correspond to an irreducible local system. Assume that there exists a nonzero Hermitian form $H$ on $\mathbb{C}^{n}$ invariant under the group $\Gamma=\left\langle T_{1}, \ldots, T_{k}\right\rangle$, i.e.,

$$
T_{s}^{*} H T_{s}=H, \quad s=1, \ldots, k
$$

Since $\operatorname{ker}(H)$ is invariant under all $T_{s}$ by irreducibility, we get that any such $H$ must be non-degenerate. This implies, in particular, that $\left(T_{s}{ }^{*}\right)^{-1}$ and $T_{s}$ are conjugate. Therefore, the sets of eigenvalues of $T_{s}$ are invariant under the map $z \mapsto \bar{z}^{-1}$. This is certainly the case if the eigenvalues are in the unit circle.

On the other hand, if the eigenvalues of $T_{s}$ are invariant under the map $z \mapsto \bar{z}^{-1}$ for all $s$ then the $\left(T_{s}^{*}\right)^{-1}$ give another solution to (1). If our system is rigid, then there exists $H$ satisfying (6). Up to a possible scalar factor $H$ is a Hermitian form invariant under the monodromy group.

The set $\mathbb{T}^{\text {irr }}$ has finitely many connected components. The signature of $H$ is constant on these components as it is continuous with integer values. We may further break the symmetry and choose the exponents satisfying $\alpha_{1}<\alpha_{2}$ and $\gamma_{1}<\gamma_{2}<\gamma_{3}<\gamma_{4}$ (recall that $\exp \left(2 \pi i \alpha_{j}\right)=a_{j}$ and so on). Then we find that there is a unique connected component where $H$ is positive definite.

It is worth noting that (6) is a system of linear equations in the entries of $H$ and can be easily solved. More generally, if $\left\{A_{k}\right\}$ and $\left\{B_{k}\right\}$ are two collections of matrices, then we can easily test if they are simultaneously conjugate by solving the system $A_{k} X-X B_{k}=0$. In our computations with monodromy groups, we often rely on this observation.

We can compute the invariant form explicitly starting from (3). Equation (4) implies in this case that $A, D$, and $B C$ are real. After making a suitable conjugation for $(B, C)$, we may assume that $A, B, C, D$ are real numbers. The invariant Hermitian matrix is then

$$
H=(A D-B C)\left(\begin{array}{cccc}
C(1-D E) & B C E & C(1-D) & B C \\
B C E & B(1-A E) & B C & B(1-A) \\
C(1-D) & B C & C(1-D) & B C \\
B C & B(1-A) & B C & B(1-A)
\end{array}\right)
$$

where $E=(A+D-1) /(A D-B C)$. The determinant of $H$ is

$$
(B C)^{2}(A D-B C-A-D+1)^{3}(A D-B C)^{3} .
$$

Using (7), we can easily describe $\mathbb{T}_{+}^{\text {irr }}$ in terms of the parameters $(A, D, t)$ where $t=B C$. If we look at the connected components of the set $\mathbb{R}^{3} \backslash V^{\prime}$, where $V^{\prime}=\{(A, D, t) \mid t(t-$ $A D)(t-(1-A)(1-D))=0\}$, and compute the signature in each case, we find that $H$ is positive definite if and only if

$$
\left\{\begin{array}{l}
0<A, D<1 \\
0<B C<A D \\
0<B C<(1-A)(1-D)
\end{array}\right.
$$

To derive a criterion in terms of eigenvalues requires more work, but can be done similarly. The final criterion is then the following. Let $\mathcal{I}_{1}$ be the open $\operatorname{arc}$ in $S^{1}$ with end points 0 , and $b^{-1}$ (any of the two possibilities), and let $\mathcal{I}_{2}$ be the arc with end points 
$\left(b^{-1} a_{1}^{-1}, b^{-1} a_{2}^{-1}\right)$, where among the two arcs we pick the one that contains the point $b^{-1}$.

Proposition 2 The invariant Hermitian form $H$ is definite if and only if for some labeling $c_{1}, \ldots, c_{4}$ of the eigenvalues of $T_{\infty}$ we have

(i) $c_{1}, c_{2} \in \mathcal{I}_{1}, \quad c_{3}, c_{4} \notin \mathcal{I}_{1}$,

(ii) $c_{1} c_{2}, c_{3} c_{4}, c_{1} c_{3}, c_{2} c_{4} \in \mathcal{I}_{2}, \quad c_{1} c_{4}, c_{2} c_{3} \notin \mathcal{I}_{2}$.

\subsection{Integrality}

The matrices given by Goursat (3), when expressed in terms of the eigenvalues, have nontrivial denominators. On the other hand, as discussed in the introduction, we should be able to exhibit the monodromy group integrally. In particular, we should be able to find integral form of our local monodromies. Integrality is crucial to analyze the cases with finite monodromy (Sect. 5).

The first observation is that we may choose $T_{\infty}$ as the companion form of $q_{\infty}$ since it has no repeated roots. After some experimentation, we found we can choose $T_{1}$ as follows.

$$
T_{1}=\left(\begin{array}{rrrr}
\sigma_{1} & 0 & 0 & \sigma_{2}^{-1} a_{1}^{-1} \\
\sigma_{2}\left(a_{1}+1\right) & \sigma_{1} & 1 & \sigma_{1} \sigma_{2}^{-1} a_{1}^{-1} \\
-\sigma_{1} \sigma_{2} a_{1} & -\sigma_{2} & 0 & 1+a_{1}^{-1} \\
-\sigma_{2}^{2} a_{1} & 0 & 0 & 0
\end{array}\right), \quad T_{\infty}=\left(\begin{array}{rrrr}
0 & 0 & 0 & -\tau_{4} \\
1 & 0 & 0 & \tau_{3} \\
0 & 1 & 0 & -\tau_{2} \\
0 & 0 & 1 & \tau_{1}
\end{array}\right),
$$

where $\sigma_{i}=e_{i}\left(b_{1}, b_{2}\right), \tau_{i}=e_{i}\left(c_{1}, c_{2}, c_{3}, c_{4}\right)$ are the elementary symmetric functions.

With these, using that

$$
a_{1} a_{2} \sigma_{2}^{2} \tau_{4}=1
$$

obtained by taking determinants in $T_{0} T_{1} T_{\infty}=I_{4}$, we get

$$
T_{0}=\left(\begin{array}{rrrr}
\left(a_{1}+1\right) & 0 & -\sigma_{2}^{-1} & a_{2}\left(\sigma_{1} \tau_{4}-\tau_{3}\right) \\
-\sigma_{1} a_{1} & 1 & \sigma_{1} \sigma_{2}^{-1} & a_{2}\left(\tau_{2}-\sigma_{2} \tau_{4}\right)-\sigma_{2}^{-1} \\
\sigma_{2} a_{1} & 0 & 0 & -a_{2} \tau_{1}+\sigma_{1} \sigma_{2}^{-1} \\
0 & 0 & 0 & a_{2}
\end{array}\right) .
$$

The trace field is generically given by $K=\mathbb{Q}\left(\sigma_{1}, \sigma_{2}, \tau_{1}, \ldots, \tau_{4}\right)$ and we see that we can always take as field of definition the quadratic extension $F:=K\left(a_{1}\right)$. Note that we also have $\operatorname{tr}\left(T_{0}\right)=2+a_{1}+a_{2} \in K$. Hence $a_{1}$ and $a_{2}$ are conjugate over $K$.

In fact, the local monodromies are definable over the ring $R\left[a_{1}\right]$, where $R:=$ $\mathbb{Z}\left[\sigma_{1}, \sigma_{2}, \tau_{1}, \ldots, \tau_{4}, \sigma_{2}^{-1}, \tau_{4}^{-1}\right]$ and hence the group $\Gamma$ they generate as well. The traces of all elements of the monodromy group are in $R$.

In particular, in the main case of interest for this paper (the motivic case, see the Introduction) the characteristic polynomials $q_{0}, q_{1}, q_{\infty}$ will have only roots of unity as roots. In this case, $K$ is a cyclotomic field. We conclude that the monodromy can be conjugated to lie in $\mathrm{GL}_{4}\left(\mathcal{O}_{F}\right)$, where $\mathcal{O}_{F}$ is the ring of integers of $F=K\left(a_{1}\right)$. This is consistent with the rigid local system being motivic.

For example, consider $q_{0}=(x-1)^{2}\left(x^{2}+1\right), q_{1}=\left(x^{2}-1\right)^{2}, q_{\infty}=x^{4}+\left(\zeta_{12}^{3}-\zeta_{12}\right) x^{3}-$ $\zeta_{12} x+1$, where $\zeta_{12}$ is a primitive 12-root of unity. This corresponds to row \#3 in Table 2 . 
Then our choice gives

$$
T_{0}:=\left(\begin{array}{cccc}
\zeta_{12}^{3}+1 & 0 & 1 & \zeta_{12}^{2}-1 \\
0 & 1 & 0 & -\zeta_{12}^{3}+1 \\
-\zeta_{12}^{3} & 0 & 0 & \zeta_{12}^{2} \\
0 & 0 & 0 & -\zeta_{12}^{3}
\end{array}\right), \quad T_{1}:=\left(\begin{array}{cccc}
0 & 0 & 0 & \zeta_{12}^{3} \\
\zeta_{12}^{3}+1 & 0 & 1 & 0 \\
0 & 1 & 0 & -\zeta_{12}^{3}+1 \\
-\zeta_{12}^{3} & 0 & 0 & 0
\end{array}\right)
$$

and

$$
T_{\infty}:=\left(\begin{array}{cccc}
0 & 0 & 0 & -1 \\
1 & 0 & 0 & \zeta_{12} \\
0 & 1 & 0 & 0 \\
0 & 0 & 1 & -\zeta_{12}^{3}+\zeta_{12}
\end{array}\right)
$$

These matrices generate a group $\Gamma$ of order 103680, which is a non-split central extension by $C_{4}$ of the simple group $\Gamma_{25920}$. We see here a phenomenon that occurs frequently in our examples. The quotient $\Gamma / Z(\Gamma)$ has no irreducible representation of degree 4 (the smallest non-trivial irreducible representation is of order 5), whereas a central extension, namely $\Gamma$, does.

It follows from the above discussion that for G-II cases the cocycle obstruction of Sect. 3 is generically of order dividing $2=[F: K]$. We can easily compute the corresponding matrix $X_{\sigma}$ for $\sigma$ the generator of $\operatorname{Gal}(F / K)$ as in Sect. 3. The problem is linear: we solve $T_{s} X_{\sigma}=X_{\sigma} T_{s}^{\sigma}$ generically, where $\sigma\left(a_{1}\right)=a_{2}$. We find

$$
X_{\sigma} X_{\sigma}^{\sigma}=\mu I_{4}
$$

where $\mu=-\left(a_{1} \sigma_{2}\right)^{3} w_{2}\left(q_{\infty}\right)\left(a_{1}^{-1} \sigma_{2}^{-1}\right) \in K^{\times}$. Recall that $w_{2}\left(q_{\infty}\right):=\prod_{i<j}\left(T-c_{i} c_{j}\right)$. The cocycle can be represented by a quaternion algebra. Explicitly, this is the quaternion algebra $\left(\frac{D, \mu}{K}\right)$, where $D=\operatorname{disc}(F)$ and $\mu$ is as above.

\section{Finite monodromy}

We would like to describe all cases of G-II with finite monodromy. Since the monodromy is integral (Sect. 4.3), finite monodromy is equivalent to the invariant Hermitian form being definite in every complex embedding of the field of definition. (This is the same argument used in [2].) These cases are those where all solutions to the corresponding differential equation (Sect. 7) are algebraic. Checking definiteness is easily done using the combinatorial criterion of Proposition 2, which involves the relative position of the eigenvalues of the local monodromies.

Apart from the infinitely many imprimitive cases discussed later in Sect. 12.5, the only examples of irreducible cases with finite monodromy that we obtained after an extensive search are those given in Tables 1, 2, 3 and 4.

\subsection{Description of the tables}

For each choice of eigenvalues, we list the order of the monodromy $\Gamma \subseteq \mathrm{GL}_{4}(\mathbb{C})$, an identification of $A$ and the quotient of $\Gamma / A$ using standard notation ( $A$ denotes a maximal abelian normal subgroup of $\Gamma$ ), the order of the center of $\Gamma$ and whether $\Gamma$ acts primitively or not.

By a theorem of Jordan, there are finitely many possibilities for the quotient $\Gamma / A$. The finite groups acting in four dimensions were classified by Blichfeldt (see [11] for a modern description). The group denoted by $\Gamma_{25920}$ is a simple group. 
Table $1 \quad \alpha_{1}, \alpha_{2}=1 / 3,2 / 3$

\begin{tabular}{lllllll}
\hline & $\gamma$ & $|\Gamma|$ & $\Gamma / A$ & $A$ & $|Z(\Gamma)|$ & $\mid \mathrm{mpr}$ \\
\hline 1 & $1 / 8,3 / 8,5 / 8,7 / 8$ & 48 & $S_{4}$ & $C_{2}$ & 2 & $*$ \\
2 & $1 / 5,2 / 5,3 / 5,4 / 5$ & 60 & $A_{5}$ & 1 & 1 & \\
3 & $1 / 10,3 / 10,7 / 10,9 / 10$ & 120 & $A_{5}$ & $C_{2}$ & 2 & \\
4 & $1 / 12,5 / 12,7 / 12,11 / 12$ & 144 & $C_{2} \times A_{4}$ & $C_{6}$ & 2 & $*$ \\
5 & $1 / 20,9 / 20,13 / 20,17 / 20$ & 240 & $A_{5}$ & $C_{4}$ & 4 & \\
6 & $2 / 9,1 / 3,5 / 9,8 / 9$ & 324 & $A_{4}$ & $C_{3}^{3}$ & 1 & $*$ \\
7 & $1 / 24,7 / 24,17 / 24,23 / 24$ & 576 & $S_{4} \times A_{4}$ & $C_{2}$ & 2 & \\
8 & $1 / 28,9 / 28,3 / 4,25 / 28$ & 672 & $\mathrm{PSL}_{2}\left(\mathbb{F}_{7}\right)$ & $C_{4}$ & 4 & \\
9 & $1 / 20,9 / 20,11 / 20,19 / 20$ & 720 & $C_{2} \times A_{5}$ & $C_{6}$ & 2 & $*$ \\
10 & $1 / 15,4 / 15,11 / 15,14 / 15$ & 1440 & $A_{5} \times A_{4}$ & $C_{2}$ & 2 & \\
11 & $1 / 30,11 / 30,19 / 30,29 / 30$ & 1440 & $A_{5} \times A_{4}$ & $C_{2}$ & 2 & \\
12 & $1 / 40,9 / 40,31 / 40,39 / 40$ & 2880 & $A_{5} \times S_{4}$ & $C_{2}$ & 2 & \\
\hline
\end{tabular}

Table $2 \alpha_{1}, \alpha_{2}=1 / 4,3 / 4$

\begin{tabular}{lllllll}
\hline & $\gamma$ & $|\Gamma|$ & $\Gamma / A$ & $A$ & $|Z(\Gamma)|$ & $\mid \mathrm{mpr}$ \\
\hline 1 & $1 / 12,5 / 12,7 / 12,11 / 12$ & 192 & $C_{2} \times S_{4}$ & $C_{4}$ & 2 & $*$ \\
2 & $1 / 20,9 / 20,13 / 20,17 / 20$ & 640 & $C_{2}^{4} \rtimes D_{5}$ & $C_{4}$ & 4 & \\
3 & $1 / 36,13 / 36,25 / 36,11 / 12$ & 103680 & $\Gamma_{25920}$ & $C_{4}$ & 4 & \\
\hline
\end{tabular}

Table $3 \alpha_{1}, \alpha_{2}=1 / 5,4 / 5$

\begin{tabular}{lllllll}
\hline & $\gamma$ & $|\Gamma|$ & $\Gamma / A$ & $A$ & $|Z(\Gamma)|$ & $\mid \mathrm{mpr}$ \\
\hline 1 & $1 / 12,5 / 12,7 / 12,11 / 12$ & 1200 & $C_{2} \times A_{5}$ & $C_{10}$ & 2 & $*$ \\
2 & $2 / 15,7 / 15,8 / 15,13 / 15$ & 7200 & $A_{5} \times A_{5}$ & $C_{2}$ & 2 & \\
3 & $1 / 20,9 / 20,11 / 20,19 / 20$ & 1200 & $C_{2} \times A_{5}$ & $C_{10}$ & 2 & $*$ \\
4 & $1 / 30,11 / 30,19 / 30,29 / 30$ & 7200 & $A_{5} \times A_{5}$ & $C_{2}$ & 2 & \\
\hline
\end{tabular}

We should note that we can always twist the local monodromies by multiplying by scalars matrices so that the resulting triple is in $\mathrm{SL}_{4}(\mathbb{C})$. If the group acts primitively, the normal subgroup $A$ consists of scalars. It follows that there are finitely many possible primitive $\Gamma$ up to twisting; we will see in Sect. 12 that this is not the case for imprimitive groups.

\subsection{Special case}

We start by discussing a special, simpler case. Assume that the characteristic polynomials $q_{0}, q_{1}, q_{\infty}$ of the local monodromies at the respective singularities have real coefficients and that $q_{1}=(T-1)^{2}(T+1)^{2}$. Let $\gamma_{i} \in(0,1)$ for $i=1, \ldots, 4$ be the exponents of the roots of $q_{\infty}$ (so that $\left.c_{j}=\exp \left(2 \pi i \gamma_{j}\right)\right)$ and similarly let $\alpha_{1} \in(0,1 / 2)$ be such that the exponents of $q_{0}$ are $0,0, \alpha_{1}, 1-\alpha_{1}$.

A special case of Proposition 2 reduces in this case to the following. Let $\delta_{1}, \ldots, \delta_{6}$ be representatives in $(0,1)$ (with multiplicities) of the exponents $\gamma_{i}+\gamma_{j}$ for $i<j$. Define $n_{1}$ as the number of $\gamma_{i}$ in the interval $(0,1 / 2)$ and $n_{2}$ the number of $\delta_{i}$ (counting with multiplicities) in the interval $\left(1 / 2-\alpha_{1}, 1 / 2+\alpha_{1}\right)$.

Proposition 3 With the above assumptions and notations the invariant Hermitian form $H$ is definite if and only if $\left(n_{1}, n_{2}\right)=(2,4)$. 
Table 4 General case

\begin{tabular}{lllrllll}
\hline & $\beta, \alpha_{1}, \alpha_{2}$ & $\gamma$ & $|\Gamma|$ & $\Gamma / A$ & $A$ & $|Z(\Gamma)|$ & $\mid \mathrm{mpr}$ \\
\hline 1 & $1 / 2,1 / 2,1 / 3$ & $1 / 8,11 / 24,5 / 8,23 / 24$ & 4608 & $\left(A_{4} \times A_{4}\right) \rtimes C_{2}$ & $C_{4}^{2}$ & 4 & $*$ \\
2 & $1 / 2,1 / 2,1 / 3$ & $5 / 48,23 / 48,29 / 48,47 / 48$ & 41472 & $\left(A_{4} \times A_{4}\right) \rtimes D_{4}$ & $C_{6}^{2}$ & 6 & $*$ \\
3 & $1 / 2,1 / 2,1 / 3$ & $11 / 120,59 / 120,71 / 120,119 / 120$ & 1036800 & $\left(A_{5} \times A_{5}\right) \rtimes C_{2}$ & $C_{12}^{2}$ & 12 & $*$ \\
4 & $1 / 2,1 / 2,1 / 4$ & $7 / 48,23 / 48,31 / 48,47 / 48$ & 6144 & $C_{2}^{4} \rtimes D_{6}$ & $C_{4} \cdot C_{8}$ & 8 & $*$ \\
5 & $1 / 2,1 / 2,1 / 5$ & $7 / 40,19 / 40,27 / 40,39 / 40$ & 2880000 & $\left(A_{5} \times A_{5}\right) \rtimes C_{2}$ & $C_{20}^{2}$ & 20 & $*$ \\
6 & $1 / 2,1 / 2,1 / 5$ & $19 / 120,59 / 120,79 / 120,119 / 120$ & 2880000 & $\left(A_{5} \times A_{5}\right) \rtimes C_{2}$ & $C_{20}^{2}$ & 20 & $*$ \\
7 & $1 / 2,1 / 2,1 / 6$ & $5 / 36,17 / 36,29 / 36,11 / 12$ & 311040 & $\Gamma_{25920}$ & $C_{12}$ & 12 & \\
8 & $1 / 2,1 / 2,1 / 6$ & $11 / 60,23 / 60,47 / 60,59 / 60$ & 311040 & $\Gamma_{25920}$ & $C_{12}$ & 12 & \\
9 & $1 / 2,1 / 3,1 / 4$ & $7 / 24,5 / 12,19 / 24,11 / 12$ & 165888 & $\left(A_{4} \times A_{4}\right) \rtimes D_{4}$ & $C_{12}^{2}$ & 12 & $*$ \\
10 & $1 / 2,1 / 3,1 / 4$ & $11 / 48,23 / 48,35 / 48,47 / 48$ & 165888 & $\left(A_{4} \times A_{4}\right) \rtimes D_{4}$ & $C_{12}^{2}$ & 12 & $*$ \\
11 & $1 / 2,1 / 3,1 / 5$ & $4 / 15,7 / 15,23 / 30,29 / 30$ & 6480000 & $\left(A_{5} \times A_{5}\right) \rtimes C_{2}$ & $C_{30}^{2}$ & 30 & $*$ \\
12 & $1 / 2,1 / 3,1 / 5$ & $17 / 60,9 / 20,47 / 60,19 / 20$ & 6480000 & $\left(A_{5} \times A_{5}\right) \rtimes C_{2}$ & $C_{30}^{2}$ & 30 & $*$ \\
13 & $1 / 2,1 / 3,1 / 5$ & $19 / 60,5 / 12,49 / 60,11 / 12$ & 6480000 & $\left(A_{5} \times A_{5}\right) \rtimes C_{2}$ & $C_{30}^{2}$ & 30 & $*$ \\
14 & $1 / 2,1 / 3,1 / 5$ & $29 / 120,59 / 120,89 / 120,119 / 120$ & 6480000 & $\left(A_{5} \times A_{5}\right) \rtimes C_{2}$ & $C_{30}^{2}$ & 30 & $*$ \\
15 & $1 / 2,1 / 5,2 / 5$ & $4 / 15,13 / 30,23 / 30,14 / 15$ & 6000 & $S_{5}$ & $C_{2} \cdot C_{5}^{2}$ & 10 & $*$ \\
16 & $1 / 2,1 / 5,2 / 5$ & $9 / 40,19 / 40,29 / 40,39 / 40$ & 6000 & $S_{5}$ & $C_{2} \cdot C_{5}^{2}$ & 10 & $*$ \\
17 & $1 / 3,1 / 2,5 / 6$ & $1 / 18,7 / 18,13 / 18,5 / 6$ & 155520 & $\Gamma_{25920}$ & $C_{6}$ & 6 & \\
18 & $1 / 3,1 / 2,1 / 6$ & $5 / 18,11 / 18,5 / 6,17 / 18$ & 155520 & $\Gamma_{25920}$ & $C_{6}$ & 6 & \\
19 & $1 / 3,1 / 2,1 / 6$ & $11 / 30,17 / 30,23 / 30,29 / 30$ & 155520 & $\Gamma_{25920}$ & $C_{6}$ & 6 \\
20 & $1 / 3,1 / 3,2 / 3$ & $1 / 12,11 / 24,5 / 6,23 / 24$ & 69120 & $C_{2}^{4} \cdot A_{6}$ & $C_{12}$ & 12 \\
21 & $1 / 3,1 / 3,2 / 3$ & $2 / 15,8 / 15,11 / 15,14 / 15$ & 2160 & $A_{6}$ & $C_{6}$ & 6 & \\
22 & $1 / 3,1 / 3,2 / 3$ & $5 / 24,11 / 24,17 / 24,23 / 24$ & 2160 & $A_{6}$ & $C_{6}$ & 6 & \\
23 & $1 / 3,1 / 3,2 / 3$ & $5 / 42,17 / 42,5 / 6,41 / 42$ & 15120 & $A_{7}$ & $C_{6}$ & 6 & \\
24 & $1 / 3,1 / 3,2 / 3$ & $11 / 60,23 / 60,47 / 60,59 / 60$ & 69120 & $C_{2}^{4} \cdot A_{6}$ & $C_{12}$ & 12 & \\
\hline & & & & & & \\
\end{tabular}

To illustrate the situation, here is a picture with the position of the various roots on the unit circle in the case $\alpha_{1}, \alpha_{2}=1 / 3,2 / 3$ and $\gamma=(1 / 28,9 / 28,3 / 4,25 / 28)$. Hence $\delta=(1 / 14,3 / 14,5 / 14,9 / 14,11 / 14,13 / 14)$.

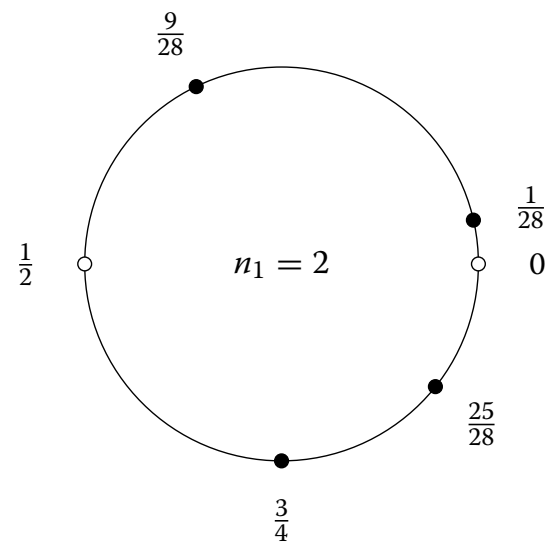




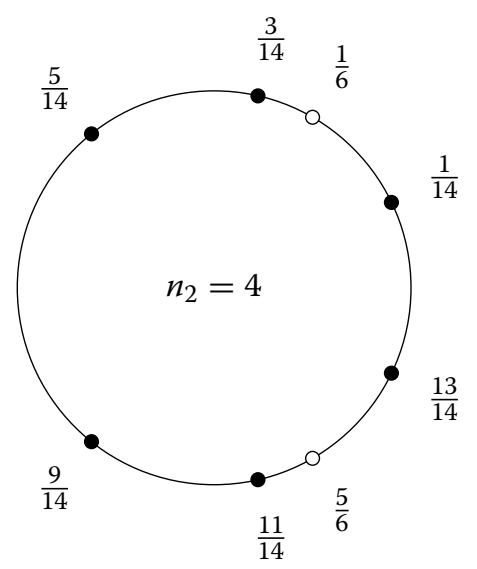

In the special case of this section, the finite monodromy cases found are listed in Tables 1 , 2 , and 3.

\subsection{General case}

Here we consider the general case (up to twisting) where the exponents are

\begin{tabular}{c|c}
$t$ & exponents \\
\hline 0 & $0,0, \alpha_{1}, \alpha_{2}$ \\
1 & $0,0, \beta, \beta$ \\
$\infty$ & $\gamma_{1}, \gamma_{2}, \gamma_{3}, \gamma_{4}$
\end{tabular}

The finite monodromy cases found are listed in Table 4.

\section{Coxeter groups}

To find explicit realizations of finite monodromy groups of G-II type, we may start with a finite group in $\mathrm{GL}_{4}(\mathbb{C})$ and attempt to build a G-II rigid local system by producing three appropriate elements $T_{0}, T_{1}, T_{\infty}$. For example, we can take a finite complex reflection group $W$ in rank 4 , hence one of the Weyl groups $A_{4}, B_{4}, F_{4}$ or the non-crystallographic case $H_{4}$. Since $T_{\infty}$ should have distinct eigenvalues different from 1 , we could start by taking $T_{\infty}$ to be a Coxeter element. Similarly, we can take $T_{1}$ to be the product of two commuting reflections in $W$. We may assume that these reflections are simple and hence correspond to two non-adjacent dots in the corresponding Dynkin diagram.

We illustrate the above procedure in one example in the case $H_{4}$ here and give several further examples defined over $\mathbb{Q}$ in Sect. 9. The Dynkin diagram is

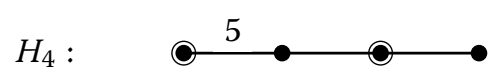

where we have circled the two chosen simple reflections. We take $T_{\infty}:=s_{1} s_{2} s_{3} s_{4}, T_{1}:=$ $s_{1} s_{3}$ and $T_{0}$ so that $T_{0} T_{1} T_{\infty}=1$.

With the help of MAGMA (see the actual calculations below), we find that

$$
T_{0}=\left(\begin{array}{rrrr}
1 & \tau & \tau & 0 \\
0 & 0 & -1 & 0 \\
0 & 0 & 1 & 1 \\
0 & -1 & -1 & -1
\end{array}\right), \quad T_{1}=\left(\begin{array}{rrrr}
-1 & 0 & 0 & 0 \\
\tau & 1 & 1 & 0 \\
0 & 0 & -1 & 0 \\
0 & 0 & 1 & 1
\end{array}\right), \quad T_{\infty}=\left(\begin{array}{rrrr}
-1 & -\tau & -\tau & -\tau \\
\tau & \tau & \tau & \tau \\
0 & 1 & 0 & 0 \\
0 & 0 & 1 & 0
\end{array}\right),
$$

where $\tau^{2}-\tau-1=0$. 
Taking the embedding into $\mathbb{R}$ where $\tau=(1+\sqrt{5}) / 2=1.618033988 \cdots$ is the golden ratio, the exponents of the local monodromies are:

$$
T_{0}:(0,0,1 / 3,2 / 3), \quad T_{1}:(0,0,1 / 2,1 / 2), \quad T_{\infty}:(1 / 30,11 / 30,19 / 30,29 / 30)
$$

and hence this example corresponds to row \#11 in the above table for $\alpha_{1}, \alpha_{2}=1 / 3,2 / 3$. This shows, in particular, that here the monodromy representation can be realized over $\mathbb{Z}[\tau]$, the ring of integers of the trace field $K:=\mathbb{Q}(\sqrt{5})$. This is consistent with our discussion in Sect. 4.3.

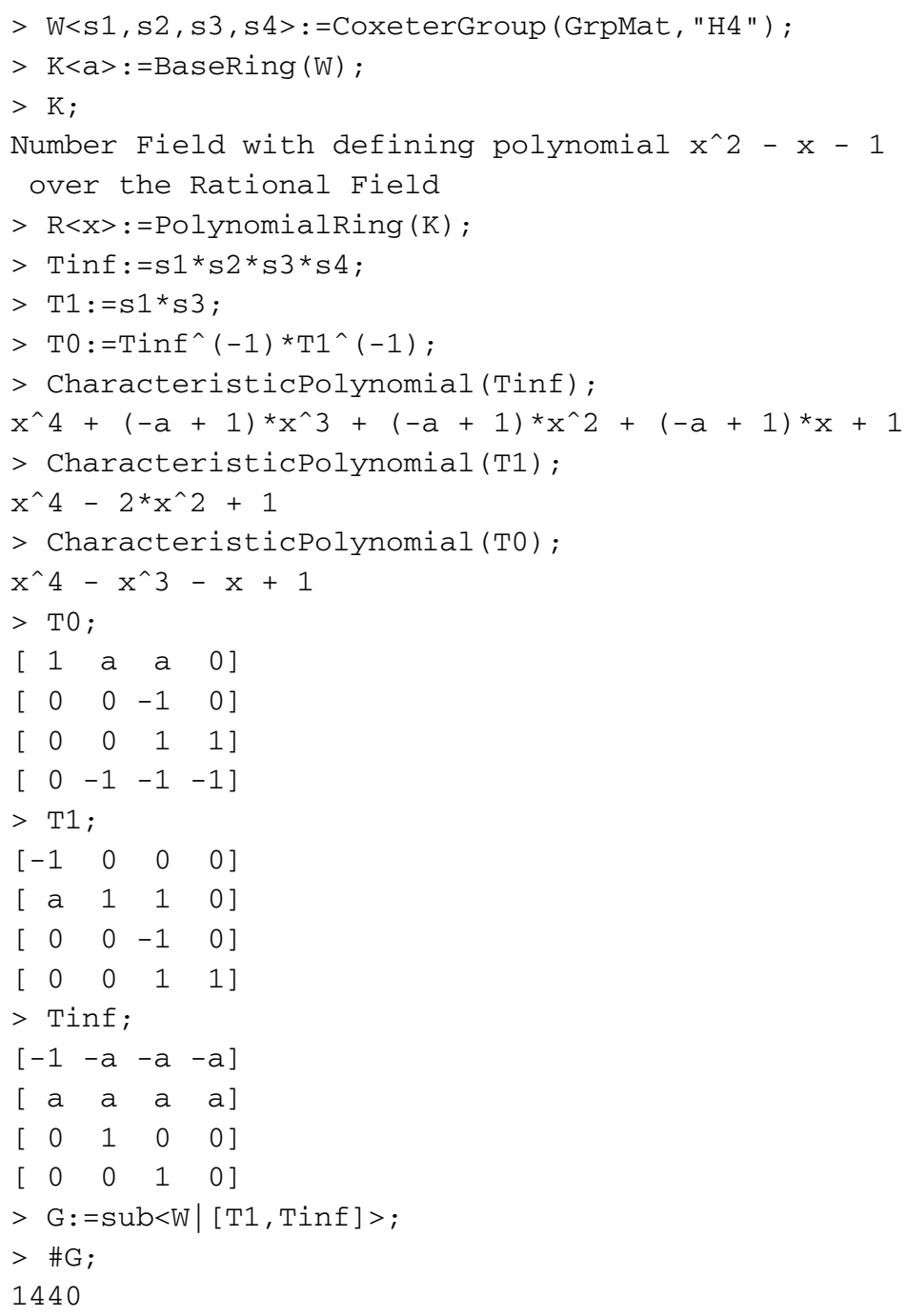

\section{Differential equation}

Goursat $[10, \$ 10]$ computes explicitly an order four linear differential equation of type G-II with given local monodromies. Let the exponents of these local monodromies be

$$
0:\left(0,1,1-\alpha_{1}, 1-\alpha_{2}\right), \quad 1:(0,1, \beta, \beta+1), \quad \infty:\left(\gamma_{1}, \gamma_{2}, \gamma_{3}, \gamma_{4}\right) .
$$

These are related by the equation

$$
\beta=\frac{1}{2}\left(1+e_{1}(\alpha)-e_{1}(\gamma)\right), \quad \alpha:=\left(\alpha_{1}, \alpha_{2}\right), \quad \gamma:=\left(\gamma_{1}, \ldots, \gamma_{4}\right),
$$

where $e_{n}(x)$ denotes the elementary symmetric functions of the quantities $x=\left(x_{1}, x_{2}, \ldots\right)$. 
The shape of the exponents forces the differential equation to be of the following form

$$
\begin{aligned}
x^{2}(x-1)^{2} \frac{d^{4}}{d x^{4}} & +(A x-B) x(x-1) \frac{d^{3}}{d x^{3}}+\left(C x^{2}-D x+E\right) \frac{d^{2}}{d x^{2}} \\
& +(F x-G) \frac{d}{d x}+H=0 .
\end{aligned}
$$

for certain constants $A, B, \ldots, H$. Imposing further that there are no logarithmic solutions at $x=1$ completely determines these constants. Following Goursat, we obtain the following values (the expression for $G$ he gives is not quite right and is here corrected.)

$$
\begin{aligned}
& A=6+e_{1}(\gamma), \\
& B=3+e_{1}(\alpha), \\
& C=7+3 e_{1}(\gamma)+e_{2}(\gamma), \\
& D=E+C-(\beta-1)(\beta-2), \\
& E=1+e_{1}(\alpha)+e_{2}(\alpha), \\
& F=1+e_{1}(\gamma)+e_{2}(\gamma)+e_{3}(\gamma), \\
& G=F+2(\beta-1)(\beta-2)(\beta-3)+(\beta-1)(\beta-2)(2 A-B)+(\beta-1)(2 C-D), \\
& H=e_{4}(\gamma) .
\end{aligned}
$$

As worked out also by Goursat $[10,(18)]$, the coefficients $a_{n}$ of a power series solution $\sum_{n \geq 0} a_{n} x^{n}$ to the differential equation satisfy the second-order recursion

$$
C_{0} a_{n}=C_{1} a_{n-1}+C_{2} a_{n-2}
$$

where

$$
\begin{aligned}
& C_{0}:=(n+1)(n+2)[n(n-1)+B n+E], \\
& C_{1}:=(n+1)[2 n(n-1)(n-2)+(A+B) n(n-1)+D n+G], \\
& C_{2}:=-[n(n-1)(n-2)(n-3)+A n(n-1)(n-2)+C n(n-1)+F n+H] .
\end{aligned}
$$

For example, if we take $\alpha=(1 / 4,3 / 4)$ and $\gamma=(1 / 5,2 / 5,3 / 5,-1 / 5)$ (so that $\beta=1 / 2$ ) we obtain the differential equation

$$
\begin{aligned}
& x^{2}(x-1)^{2} \frac{d^{4}}{d x^{4}}+x(x-1)(7 x-4) \frac{d^{3}}{d x^{3}} \\
& \quad+\left(51 / 5 x^{2}-931 / 80 x+35 / 16\right) \frac{d^{2}}{d x^{2}}+(54 / 25 x-1223 / 800) \frac{d}{d x}-6 / 625,
\end{aligned}
$$

which indeed has local exponents

$$
0:(0,1,1 / 4,3 / 4), \quad 1:(0,1 / 2,1,3 / 2), \quad \infty:(-1 / 5,1 / 5,2 / 5,3 / 5) .
$$

The first few coefficients of the power series expansion of a basis of holomorphic solutions to the differential equation at $x=0$ are as follows:

$$
\begin{aligned}
\phi_{0}= & 1+48 / 21875 x^{2}+28088 / 18046875 x^{3}+6589643 / 5865234375 x^{4} \\
& +57582020413 / 67659667968750 x^{5}+O\left(x^{6}\right), \\
\phi_{1}= & x+1223 / 3500 x^{2}+1096811 / 5775000 x^{3}+370276451 / 3003000000 x^{4} \\
& +15278570717561 / 173208750000000 x^{5}+O\left(x^{6}\right) .
\end{aligned}
$$


Table 5 Signature $(2,2)$

\begin{tabular}{|c|c|c|c|c|c|}
\hline & $\alpha_{1}, \alpha_{2}$ & $\beta_{1}, \beta_{2}$ & $\gamma_{1}, \gamma_{2}, \gamma_{3}, \gamma_{4}$ & $D$ & $\mu$ \\
\hline 1 & $1 / 3,2 / 3$ & $0,1 / 2$ & $1 / 4,1 / 3,2 / 3,3 / 4$ & -3 & -2 \\
\hline 2 & $1 / 3,2 / 3$ & $0,1 / 2$ & $1 / 6,1 / 4,3 / 4,5 / 6$ & -3 & -2 \\
\hline 3 & $1 / 3,2 / 3$ & $1 / 3,2 / 3$ & $1 / 6,1 / 4,3 / 4,5 / 6$ & -3 & -2 \\
\hline 4 & $1 / 3,2 / 3$ & $1 / 3,2 / 3$ & $1 / 10,3 / 10,7 / 10,9 / 10$ & -3 & -1 \\
\hline 5 & $1 / 3,2 / 3$ & $1 / 6,5 / 6$ & $1 / 4,1 / 3,2 / 3,3 / 4$ & -3 & -2 \\
\hline 6 & $1 / 3,2 / 3$ & $1 / 6,5 / 6$ & $1 / 5,2 / 5,3 / 5,4 / 5$ & -3 & -1 \\
\hline 7 & $1 / 4,3 / 4$ & $0,1 / 2$ & $1 / 4,1 / 3,2 / 3,3 / 4$ & -4 & -3 \\
\hline 8 & $1 / 4,3 / 4$ & $0,1 / 2$ & $1 / 6,1 / 3,2 / 3,5 / 6$ & -4 & -1 \\
\hline 9 & $1 / 4,3 / 4$ & $0,1 / 2$ & $1 / 6,1 / 4,3 / 4,5 / 6$ & -4 & -3 \\
\hline 10 & $1 / 4,3 / 4$ & $0,1 / 2$ & $1 / 5,2 / 5,3 / 5,4 / 5$ & -4 & -1 \\
\hline 11 & $1 / 4,3 / 4$ & $0,1 / 2$ & $1 / 10,3 / 10,7 / 10,9 / 10$ & -4 & -1 \\
\hline 12 & $1 / 4,3 / 4$ & $1 / 3,2 / 3$ & $1 / 6,1 / 4,3 / 4,5 / 6$ & -4 & -3 \\
\hline 13 & $1 / 4,3 / 4$ & $1 / 3,2 / 3$ & $1 / 10,3 / 10,7 / 10,9 / 10$ & -4 & -1 \\
\hline 14 & $1 / 4,3 / 4$ & $1 / 3,2 / 3$ & $1 / 12,5 / 12,7 / 12,11 / 12$ & -4 & 1 \\
\hline 15 & $1 / 4,3 / 4$ & $1 / 4,3 / 4$ & $1 / 12,5 / 12,7 / 12,11 / 12$ & -4 & 1 \\
\hline 16 & $1 / 4,3 / 4$ & $1 / 6,5 / 6$ & $1 / 4,1 / 3,2 / 3,3 / 4$ & -4 & -3 \\
\hline 17 & $1 / 4,3 / 4$ & $1 / 6,5 / 6$ & $1 / 5,2 / 5,3 / 5,4 / 5$ & -4 & -1 \\
\hline 18 & $1 / 4,3 / 4$ & $1 / 6,5 / 6$ & $1 / 12,5 / 12,7 / 12,11 / 12$ & -4 & 1 \\
\hline 19 & $1 / 6,5 / 6$ & $0,1 / 2$ & $1 / 4,1 / 3,2 / 3,3 / 4$ & -3 & -2 \\
\hline 20 & $1 / 6,5 / 6$ & $0,1 / 2$ & $1 / 6,1 / 3,2 / 3,5 / 6$ & -3 & -2 \\
\hline 21 & $1 / 6,5 / 6$ & $0,1 / 2$ & $1 / 6,1 / 4,3 / 4,5 / 6$ & -3 & -2 \\
\hline 22 & $1 / 6,5 / 6$ & $0,1 / 2$ & $1 / 5,2 / 5,3 / 5,4 / 5$ & -3 & -1 \\
\hline 23 & $1 / 6,5 / 6$ & $0,1 / 2$ & $1 / 8,3 / 8,5 / 8,7 / 8$ & -3 & -1 \\
\hline 24 & $1 / 6,5 / 6$ & $0,1 / 2$ & $1 / 10,3 / 10,7 / 10,9 / 10$ & -3 & -1 \\
\hline 25 & $1 / 6,5 / 6$ & $1 / 3,2 / 3$ & $1 / 6,1 / 4,3 / 4,5 / 6$ & -3 & -2 \\
\hline 26 & $1 / 6,5 / 6$ & $1 / 3,2 / 3$ & $1 / 5,2 / 5,3 / 5,4 / 5$ & -3 & 1 \\
\hline 27 & $1 / 6,5 / 6$ & $1 / 3,2 / 3$ & $1 / 8,3 / 8,5 / 8,7 / 8$ & -3 & 1 \\
\hline 28 & $1 / 6,5 / 6$ & $1 / 3,2 / 3$ & $1 / 10,3 / 10,7 / 10,9 / 10$ & -3 & 1 \\
\hline 29 & $1 / 6,5 / 6$ & $1 / 3,2 / 3$ & $1 / 12,5 / 12,7 / 12,11 / 12$ & -3 & 2 \\
\hline 30 & $1 / 6,5 / 6$ & $1 / 4,3 / 4$ & $1 / 5,2 / 5,3 / 5,4 / 5$ & -3 & 1 \\
\hline 31 & $1 / 6,5 / 6$ & $1 / 4,3 / 4$ & $1 / 8,3 / 8,5 / 8,7 / 8$ & -3 & 1 \\
\hline 32 & $1 / 6,5 / 6$ & $1 / 4,3 / 4$ & $1 / 10,3 / 10,7 / 10,9 / 10$ & -3 & 1 \\
\hline 33 & $1 / 6,5 / 6$ & $1 / 4,3 / 4$ & $1 / 12,5 / 12,7 / 12,11 / 12$ & -3 & 2 \\
\hline 34 & $1 / 6,5 / 6$ & $1 / 6,5 / 6$ & $1 / 4,1 / 3,2 / 3,3 / 4$ & -3 & -2 \\
\hline 35 & $1 / 6,5 / 6$ & $1 / 6,5 / 6$ & $1 / 5,2 / 5,3 / 5,4 / 5$ & -3 & 1 \\
\hline 36 & $1 / 6,5 / 6$ & $1 / 6,5 / 6$ & $1 / 8,3 / 8,5 / 8,7 / 8$ & -3 & 1 \\
\hline 37 & $1 / 6,5 / 6$ & $1 / 6,5 / 6$ & $1 / 10,3 / 10,7 / 10,9 / 10$ & -3 & 1 \\
\hline 38 & $1 / 6,5 / 6$ & $1 / 6,5 / 6$ & $1 / 12,5 / 12,7 / 12,11 / 12$ & -3 & 2 \\
\hline
\end{tabular}

As expected from a motivic situation the denominators of the coefficients appear to grow only exponentially, rather than what could be expected generically from solutions to recursion (9).

\section{Field of moduli $\mathbb{Q}$}

It is easy to list all cases of irreducible G-II rigid local systems with field of moduli $\mathbb{Q}$ as there are only finitely many cyclotomic polynomials of fixed degree and with coefficients in $\mathbb{Q}$. We list the results in Tables 5 and 6 according to the signature of the respective Hermitian form. 
Table 6 Signature $(4,0)$

\begin{tabular}{lllllr}
\hline & $\alpha_{1}, \alpha_{2}$ & $\beta_{1}, \beta_{2}$ & $\gamma_{1}, \gamma_{2}, \gamma_{3}, \gamma_{4}$ & $D$ & $\mu$ \\
\hline 1 & $1 / 3,2 / 3$ & $0,1 / 2$ & $1 / 5,2 / 5,3 / 5,4 / 5$ & -3 & 1 \\
2 & $1 / 3,2 / 3$ & $0,1 / 2$ & $1 / 8,3 / 8,5 / 8,7 / 8$ & -3 & 1 \\
3 & $1 / 3,2 / 3$ & $0,1 / 2$ & $1 / 10,3 / 10,7 / 10,9 / 10$ & -3 & 1 \\
4 & $1 / 3,2 / 3$ & $0,1 / 2$ & $1 / 12,5 / 12,7 / 12,11 / 12$ & -3 & 2 \\
5 & $1 / 3,2 / 3$ & $1 / 3,2 / 3$ & $1 / 5,2 / 5,3 / 5,4 / 5$ & -3 & -1 \\
6 & $1 / 3,2 / 3$ & $1 / 3,2 / 3$ & $1 / 8,3 / 8,5 / 8,7 / 8$ & -3 & -1 \\
7 & $1 / 3,2 / 3$ & $1 / 4,3 / 4$ & $1 / 6,1 / 3,2 / 3,5 / 6$ & -3 & -2 \\
8 & $1 / 3,2 / 3$ & $1 / 4,3 / 4$ & $1 / 5,2 / 5,3 / 5,4 / 5$ & -3 & -1 \\
9 & $1 / 3,2 / 3$ & $1 / 4,3 / 4$ & $1 / 8,3 / 8,5 / 8,7 / 8$ & -3 & -1 \\
10 & $1 / 3,2 / 3$ & $1 / 4,3 / 4$ & $1 / 10,3 / 10,7 / 10,9 / 10$ & -3 & -1 \\
11 & $1 / 3,2 / 3$ & $1 / 6,5 / 6$ & $1 / 8,3 / 8,5 / 8,7 / 8$ & -3 & -1 \\
12 & $1 / 3,2 / 3$ & $1 / 6,5 / 6$ & $1 / 10,3 / 10,7 / 10,9 / 10$ & -3 & -1 \\
13 & $1 / 4,3 / 4$ & $0,1 / 2$ & $1 / 12,5 / 12,7 / 12,11 / 12$ & -4 & 1 \\
14 & $1 / 4,3 / 4$ & $1 / 3,2 / 3$ & $1 / 5,2 / 5,3 / 5,4 / 5$ & -4 & -1 \\
15 & $1 / 4,3 / 4$ & $1 / 4,3 / 4$ & $1 / 6,1 / 3,2 / 3,5 / 6$ & -4 & -1 \\
16 & $1 / 4,3 / 4$ & $1 / 4,3 / 4$ & $1 / 5,2 / 5,3 / 5,4 / 5$ & -4 & -1 \\
17 & $1 / 4,3 / 4$ & $1 / 4,3 / 4$ & $1 / 10,3 / 10,7 / 10,9 / 10$ & -4 & -1 \\
18 & $1 / 4,3 / 4$ & $1 / 6,5 / 6$ & $1 / 10,3 / 10,7 / 10,9 / 10$ & -4 & -1 \\
\hline
\end{tabular}

Table 7 Quaternion algebras

\begin{tabular}{llllll}
\hline$D \backslash \mu$ & -3 & -2 & -1 & 1 & 2 \\
\hline-3 & {$[3, \infty]$} & {$[2, \infty]$} & {$[3, \infty]$} & {[]} & {$[2,3]$} \\
-4 & {$[3, \infty]$} & {$[2, \infty]$} & {$[2, \infty]$} & {[]} & \\
\hline
\end{tabular}

Recall that the obstruction for the realizability of the monodromy group over the field of moduli is given by the quaternion algebra $\left(\frac{D, \mu}{K}\right)$, where $D=\operatorname{disc}(F)$, with $F=\mathbb{Q}\left(a_{1}\right)$, and $\mu$ is the number computed in (8). There are only four different quaternion algebras over $\mathbb{Q}$ that appear depending on $\alpha_{1}, \alpha_{2}$. To give these algebras is enough to give the list $\left[p_{1}, \ldots, p_{2 r}\right]$ of ramified primes. These are: [] (i.e., the matrix algebra), $[2, \infty],[3, \infty]$ or $[2,3]$ (see Table 7).

\section{Finite monodromy $K=\mathbb{Q}$}

As shown in Table 6, there are only four cases of finite monodromy with field of moduli $\mathbb{Q}$ that can be realized over $\mathbb{R}$ (rows \#1,\#2,\#4, and \#14; note that \#3 is a twist of \#1). Three cases are actually definable over $\mathbb{Q}$; we list these first. We give the fourth case in Sect. 9.4; it has the quaternion algebra ramified at $[2,3]$ as an obstruction and is hence not definable over $\mathbb{Q}$.

We will construct these monodromy groups as subgroups in Coxeter groups as in Sect. 6; we circle in the corresponding Dynkin diagram the two chosen simple reflections.

$9.1(1 / 3,2 / 3),(0,1 / 2),(1 / 5,2 / 5,3 / 5,4 / 5)$

We can find this case as a subgroup of $S_{5}$ viewed as the Coxeter group of the root system $A_{4}$.

$$
A_{4}:
$$

The monodromy group is isomorphic to $A_{5}$, the alternating group in five letters, acting in its standard representation. Here is a calculation using MAGMA. 


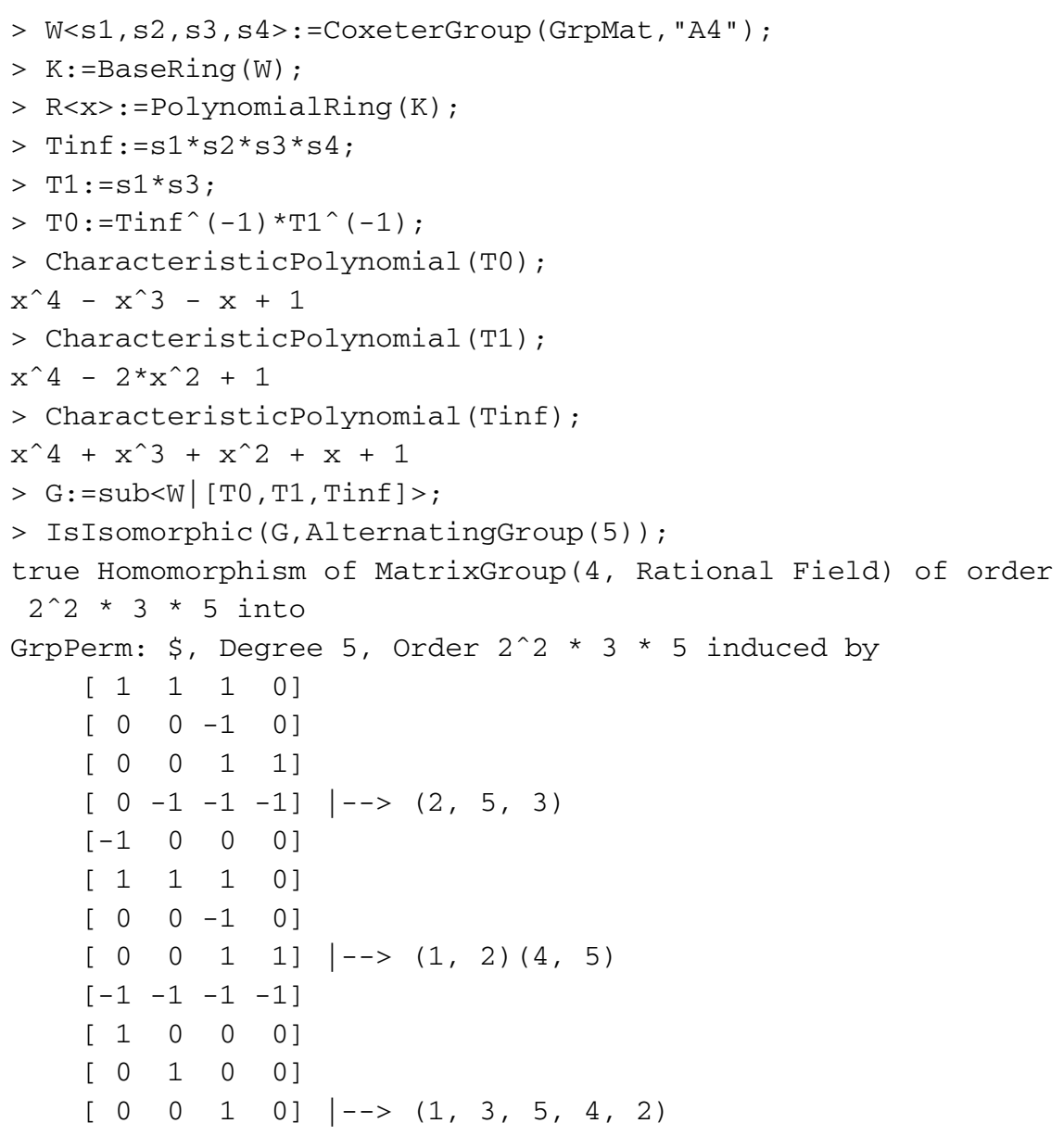

Choosing the parameters in Goursat's differential equation (Sect. 7) as

$$
\left(\alpha_{1}, \alpha_{2}\right)=(1 / 3,2 / 3) ; \quad \beta=1 / 2 ; \quad\left(\gamma_{1}, \gamma_{2}, \gamma_{3}, \gamma_{4}\right)=(1 / 5,2 / 5,-2 / 5,4 / 5)
$$

we obtain $7,4,10,413 / 36,20 / 9,46 / 25,2387 / 1800,-16 / 625$ for the eight constants $A, B, \ldots, H$. Then all holomorphic solutions at $x=0$ have power series expansion, since they represent algebraic functions of $x$, with integral coefficients, up to the power of some constant $N$. (The minimal such $N$ is called the Eisenstein constant of the algebraic function; in this example it seems to only involve the primes 2,5 and 11.)

The holomorphic solution to equation holomorphic at $x=0$ and starting as

$$
y:=1-387 / 1300 x-172773 / 2080000 x^{2}-141382989 / 3328000000 x^{3}+O\left(x^{4}\right)
$$

satisfies an algebraic equation of degree 10 over $\mathbb{Q}(x)$. (The series can be computed using the explicit form of the differential equation given by Goursat, see Sect. 7.)

The solution over $K:=\mathbb{Q}(\sqrt{-15})$ that starts as

$$
1-(123 / 475+33 / 1900 \omega) x-(271713 / 3800000+78771 / 15200000 \omega) x^{2}+O\left(x^{3}\right)
$$

where $\omega^{2}-\omega+4=0$ is a generator of the ring of integers of $K$ on the other hand, satisfies the following degree five equation

$$
P(x, y):=y^{5}+a_{3}(x) y^{3}+a_{2}(x) y^{2}+a_{1}(x) y+a_{0}(x)=0,
$$


where

$$
\begin{aligned}
a_{3}:= & 605 / 8664-715 / 2888 \omega \\
a_{2}:= & -1189825 / 2963088+70525 / 329232 \omega, \\
a_{1}:= & (298150 / 390963-11050 / 130321 \omega) x \\
& -518989705 / 900778752+19234735 / 300259584 \omega, \\
a_{0}:= & -(453252 / 2476099+151020 / 2476099 \omega) x^{2} \\
& +(3663787 / 14856594+406915 / 4952198 \omega) x \\
& -(82982887 / 900778752+9216415 / 300259584 \omega) .
\end{aligned}
$$

$9.2(1 / 4,3 / 4),(0,1 / 2),(1 / 8,3 / 8,5 / 8,7 / 8)$

We can find this case as a subgroup of the Coxeter group of the root system $B_{4}$.

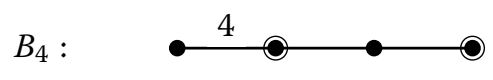

The monodromy group is isomorphic to $\mathrm{GL}_{2}\left(\mathbb{F}_{3}\right)$, of order 48 , in its unique faithful irreducible representation of dimension four. Here is a calculation using MAGMA.

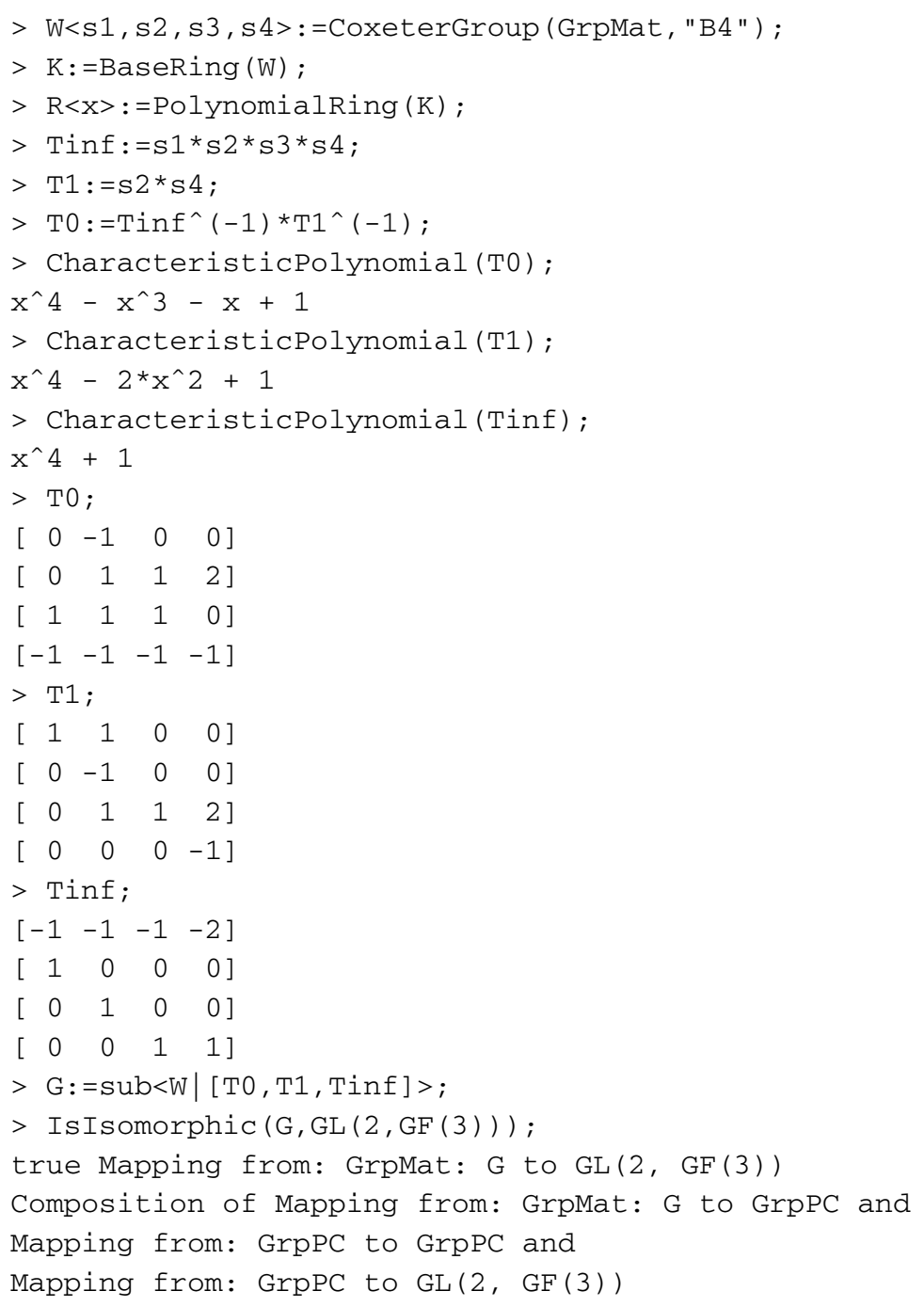


We can change basis so that $T_{\infty}$ is the companion matrix of $\Phi_{8}=T^{4}+1$. We obtain the following triple:

$$
T_{0}=\left(\begin{array}{rrrr}
0 & 1 & 1 & 0 \\
-1 & 0 & -1 & 0 \\
0 & -1 & 0 & 0 \\
0 & 1 & 0 & 1
\end{array}\right), \quad T_{1}=\left(\begin{array}{rrrr}
-1 & -1 & -1 & -1 \\
0 & 0 & 0 & -1 \\
0 & 1 & 0 & 1 \\
-1 & 0 & 0 & 1
\end{array}\right), \quad T_{\infty}=\left(\begin{array}{rrrr}
0 & 0 & 0 & -1 \\
1 & 0 & 0 & 0 \\
0 & 1 & 0 & 1 \\
0 & 0 & 1 & 0
\end{array}\right)
$$

Choosing the parameters in Goursat's differential equation below (Sect. 7) as

$$
\left(\alpha_{1}, \alpha_{2}\right)=(1 / 4,3 / 4) ; \quad \beta=1 / 2 ; \quad\left(\gamma_{1}, \gamma_{2}, \gamma_{3}, \gamma_{4}\right)=(1 / 8,3 / 8,-3 / 8,7 / 8)
$$

we obtain 7, 4, 319/32, 3295/288, 20/9, 117/64, 383/288, $-63 / 4096$ for the eight constants $A, B, \ldots, H$. Then all holomorphic solutions at $x=0$ have power series expansion with integral coefficients up to powers of 2 and 5.

The holomorphic solution to equation holomorphic at $x=0$ and starting as

$$
1+(5 / 256 \sqrt{-8}-29 / 128) x+(383 / 65536 \sqrt{-8}-527 / 8192) x^{2}+O\left(x^{3}\right)
$$

satisfies the degree eight equation

$$
P(x, y)=y^{8}+a_{6}(x) y^{6}+a_{4}(x) y^{4}+a_{2}(x) y^{2}+a_{0}(x),
$$

where

$$
\begin{aligned}
a_{6}:= & 230 / 729 \sqrt{-8}-400 / 729, \\
a_{4}:= & (1048 / 19683 \sqrt{-8}+19984 / 19683) x \\
& -(351670 / 1594323 \sqrt{-8}+1034482 / 1594323), \\
a_{2}:= & (4842880 / 43046721 \sqrt{-8}-10078688 / 43046721) x, \\
& +(-1015591450 / 10460353203 \sqrt{-8}+1684358888 / 10460353203), \\
a_{0}:= & -(27028768 / 1162261467 \sqrt{-8}+3467632 / 1162261467) x^{3} \\
& +(172219360 / 3486784401 \sqrt{-8}+238769752 / 3486784401) x^{2} \\
& -(296048878 / 10460353203 \sqrt{-8}+1067187679 / 10460353203) x \\
& +(22649710 / 10460353203 \sqrt{-8}+382087111 / 10460353203) .
\end{aligned}
$$

In addition, let $\phi_{0}:=1+0 \cdot x+O\left(x^{2}\right), \phi_{1}:=0 \cdot 1+x+O\left(x^{2}\right)$ be a basis of the holomorphic solutions to the differential equation at $x=0$ and define

$$
\psi:=\phi_{0}^{2}-891 / 16384 \phi_{1}^{2} \text {. }
$$

Then $\psi$ satisfies a degree four equation; more precisely, if $\xi$ is the hypergeometric series satisfying the trinomial equation

$$
\xi^{4}-4 \xi^{3}+27 x=0,
$$

then $\psi=-4 / 135 \xi^{3}+4 / 45 \xi^{2}+32 / 45 \xi-37 / 27$.

$9.3(1 / 4,3 / 4),(0,1 / 2),(1 / 12,5 / 12,7 / 12,11 / 12)$

We can find this case as a subgroup of the Coxeter group of the root system $F_{4}$.

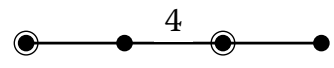


The monodromy group is isomorphic to $U_{2}\left(\mathbb{F}_{3}\right) \rtimes C_{2}$, of order 192, in one of its irreducible faithful representations of dimension four. This group is labeled $(192,988)$ in the SmallGroup database. Here is a calculation using MAGMA.

$>\mathrm{W}<\mathrm{s} 1, \mathrm{~s} 2, \mathrm{~s} 3, \mathrm{~s} 4>$ : = CoxeterGroup (GrpMat, "F4 " ) ;

$>\mathrm{K}:=\mathrm{BaseRing}(\mathrm{W})$;

$>\mathrm{R}<\mathrm{X}>$ :=PolynomialRing $(\mathrm{K})$;

$>$ Tinf: $=\mathrm{s} 1{ }^{*} \mathrm{~s} 2{ }^{*} \mathrm{~s} 3{ }^{*} \mathrm{~s} 4$;

$>\mathrm{T} 1:=\mathrm{s} 1 * \mathrm{~s} 3$;

$>\mathrm{T} 0:=\operatorname{Tinf}^{\wedge}(-1) * \mathrm{~T}^{\wedge}(-1)$;

$>$ CharacteristicPolynomial (T0);

$x^{\wedge} 4-2{ }^{*} x^{\wedge} 3+2 * x^{\wedge} 2-2 * x+1$

$>$ CharacteristicPolynomial (T1);

$\mathrm{x}^{\wedge} 4-2 * \mathrm{x}^{\wedge} 2+1$

$>$ CharacteristicPolynomial (Tinf);

$\mathrm{x}^{\wedge} 4-\mathrm{x}^{\wedge} 2+1$

$>\mathrm{TO}$;

$\left[\begin{array}{llll}1 & 1 & 2 & 0\end{array}\right]$

$\left[\begin{array}{llll}0 & 1 & 0 & 0\end{array}\right]$

$\left[\begin{array}{llll}0 & 0 & 1 & 1\end{array}\right]$

$\left[\begin{array}{llll}0 & -1 & -2 & -1\end{array}\right]$

$>\mathrm{T} 1$;

$\left[\begin{array}{cccc}-1 & 0 & 0 & 0\end{array}\right]$

$\left[\begin{array}{llll}1 & 1 & 2 & 0\end{array}\right]$

$\left[\begin{array}{llll}0 & 0 & -1 & 0\end{array}\right]$

[ $\left.\begin{array}{llll}0 & 0 & 1 & 1\end{array}\right]$

$>$ Tinf;

$\left[\begin{array}{llll}-1 & -1 & -2 & -2\end{array}\right]$

$\left[\begin{array}{llll}1 & 0 & 0 & 0\end{array}\right]$

[ $\left.\begin{array}{llll}0 & 1 & 1 & 1\end{array}\right]$

$\left[\begin{array}{llll}0 & 0 & 1 & 0\end{array}\right]$

$>\mathrm{G}:=\mathrm{sub}<\mathrm{W} \mid[\mathrm{T} 0, \mathrm{~T} 1, \mathrm{Tinf}]>$;

$>$ IdentifyGroup (G) ;

$<192$, 988>

Conjugating so that $T_{\infty}$ is the companion matrix of $\Phi_{12}=T^{4}-T^{2}+1$ we obtain the following triple

$$
T_{0}=\left(\begin{array}{rrrr}
1 & 0 & 0 & -1 \\
0 & 1 & 0 & 1 \\
0 & 0 & 1 & 1 \\
0 & -1 & -1 & -1
\end{array}\right), \quad T_{1}=\left(\begin{array}{rrrr}
0 & 1 & 1 & 1 \\
1 & 0 & 0 & -1 \\
0 & 0 & -1 & 0 \\
0 & 0 & 1 & 1
\end{array}\right), \quad T_{\infty}=\left(\begin{array}{rrrr}
0 & 0 & 0 & -1 \\
1 & 0 & 0 & 0 \\
0 & 1 & 0 & 1 \\
0 & 0 & 1 & 0
\end{array}\right) .
$$

The permutation representation of the smallest degree for this monodromy group is of degree 24. Hence some solution to the differential equation satisfies a degree 24 equation with coefficients in some number field but we have not attempted to find it.

$9.4(1 / 3,2 / 3),(0,1 / 2),(1 / 12,5 / 12,7 / 12,11 / 12)$

As already mentioned in this case, the quaternion algebra is ramified at [2,3]. As it happens, since 2 and 3 are both inert in $F=\mathbb{Q}(\sqrt{5})$, the obstruction cocycle $\xi$ becomes trivial in $F$. Therefore, by Proposition 3, we should be able to realize this case over $F$.

This is indeed the case and we can realize it again using Coxeter groups, namely as a subgroup of the non-crystallographic $W\left(H_{4}\right)$ of order 14400 . Note, however, that in this 
case $T_{\infty}$ is not a Coxeter element; we still take $T_{1}$ as a product of two commuting simple reflections.

Here are some of the computations in MAGMA.

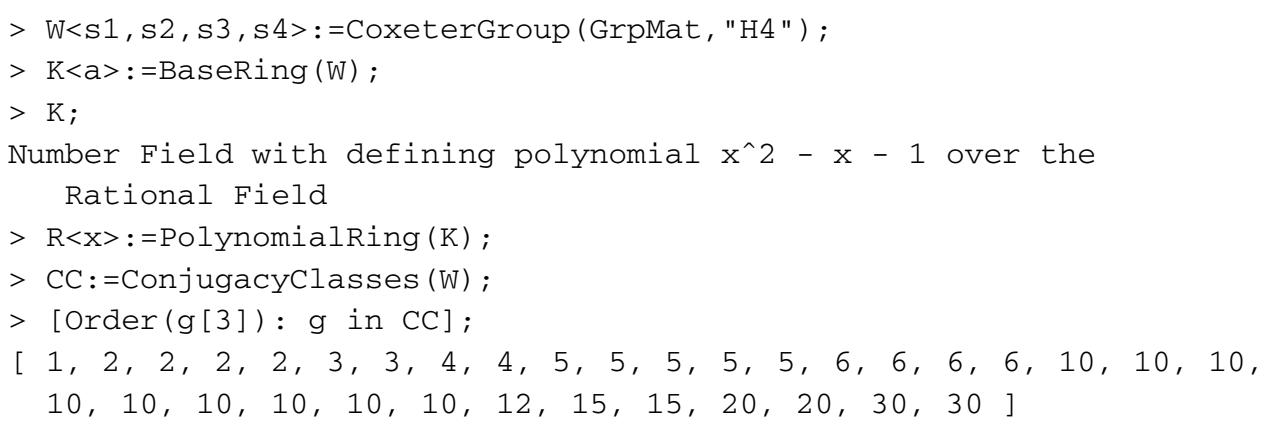

We see that there is a unique conjugacy class in $W\left(H_{4}\right)$ of order 12 . The class has 1200 elements.

$>\mathrm{C}:=$ Conjugates $(\mathrm{W}, \mathrm{CC}[28][3])$;

$>\# C$;

1200

We set $T_{1}:=s_{1} s_{3}$ and look for an element $T_{\infty}$ of order 12 such that $s_{1} s_{3} T_{\infty}$ has characteristic polynomial $T^{4}-T^{3}-T+1=(T-1)^{2}\left(T^{2}+T+1\right)$. There are a fair number of such elements; we select for example:

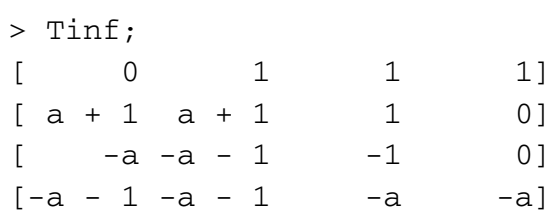

We can now construct the whole triple and compute the order of the group they generate.

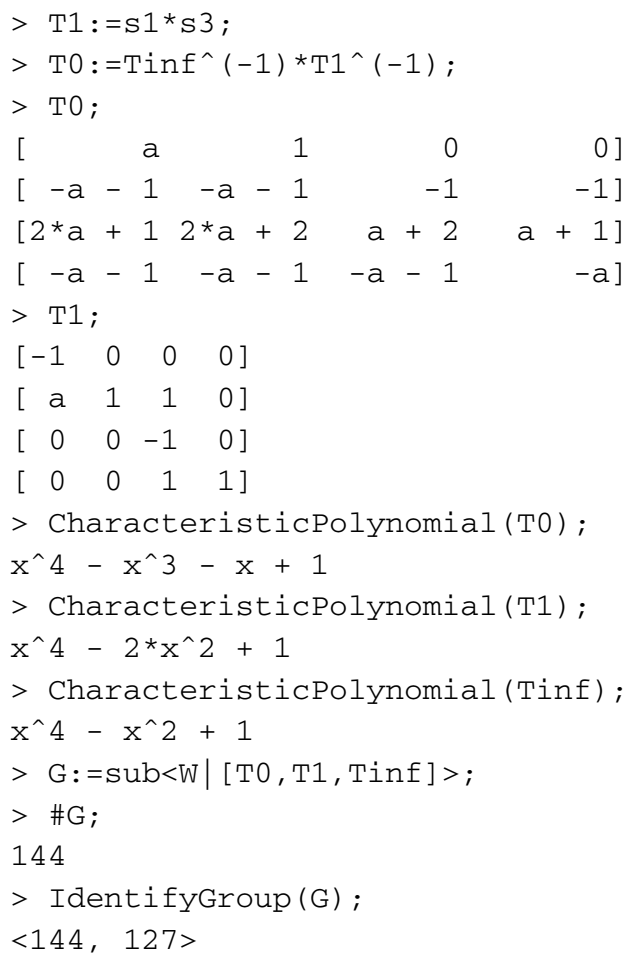


The monodromy group is then isomorphic to $\mathrm{SL}_{2}\left(\mathbb{F}_{3}\right) \rtimes S_{3}$ acting via its four-dimensional faithful irreducible representation with rational character. Note that this representation has Schur index 2 matching our obstruction calculation. Its smallest permutation representation is of degree 48 .

\section{Hurwitz example}

Some examples of G-II systems arise from modular functions. We give an example due to Hurwitz [15]. For more details on the associated Klein curve, see [7]; for general facts about modular forms, see [28].

Consider the modular function $u_{7}(\tau):=\eta(7 \tau) / \eta(\tau)$, where $\eta(\tau)$ is Dedekind's etafunction. It is known that $u_{7}^{4}$ is a Hauptmodul for $X_{1}(7)$. As a function of $t:=1728 / j$, where $j$ is the standard elliptic $j$-invariant, $u_{7}$ satisfies the algebraic equation

$$
t\left(49 u_{7}^{8}+13 u_{7}^{4}+1\right)\left(7^{4} u_{7}^{8}+245 u_{7}^{4}+1\right)^{3}-1728 u_{7}^{4}=0 .
$$

It also satisfies a fourth-order differential equation of type G-II in terms of $x=t^{-1}$

$$
\begin{aligned}
& x^{2}(x-1)^{2} \frac{d^{4} u_{7}}{d x^{4}}+x(x-1)(7 x-4) \frac{d^{3} u_{7}}{d x^{3}}+\left(573 / 56 x^{2}-5899 / 504 x+20 / 9\right) \frac{d^{2} u_{7}}{d x^{2}} \\
& +(12297 / 5488 x-39779 / 24696) \frac{d u_{7}}{d x}-57 / 87808 u_{7}=0
\end{aligned}
$$

with exponents

$$
\begin{array}{r|l}
x & \text { exponents } \\
\hline 0 & 0,1 / 3,2 / 3,1 \\
1 & 0,1 / 2,1,3 / 2 \\
\infty & -1 / 28,3 / 28,1 / 4,19 / 28
\end{array} .
$$

We see that this example is a conjugate of that in row \#8 of Table 1.

Furthermore, consider the following modular functions for $\Gamma(7)$

$$
\begin{aligned}
& \mathbf{x}(\tau)=q^{23 / 84} \prod_{n \equiv \pm 4(\bmod 7)}\left(1-q^{n}\right)^{-1}, \\
& \mathbf{y}(\tau)=q^{11 / 84} \prod_{n \equiv \pm 2(\bmod 7)}\left(1-q^{n}\right)^{-1}, \\
& \mathbf{z}(\tau)=q^{-13 / 84} \prod_{n \equiv \pm 1(\bmod 7)}\left(1-q^{n}\right)^{-1} .
\end{aligned}
$$

A full basis of solutions to (12) is then $\mathbf{x y z}, \mathbf{x}^{2} \mathbf{y}, \mathbf{y}^{2} \mathbf{z}, \mathbf{z}^{2} \mathbf{x}$. Note that $u_{7}=\mathbf{x y z}$.

\section{A family of genus two curves}

In this section, we analyze in depth the G-II system in row \#35 of Table 5, which has infinite monodromy group. We show explicitly that it is motivic by matching it to a Picard-Fuchs equation of an associated family of genus two curves.

Consider the G-II rigid local system $\mathcal{G}$ with parameters

$$
\left(\alpha_{1}, \alpha_{2}\right)=(1 / 6,5 / 6), \quad\left(\beta_{1}, \beta_{2}\right)=(1 / 6,5 / 6), \quad\left(\gamma_{1}, \gamma_{2}, \gamma_{3}, \gamma_{4}\right)=(1 / 5,2 / 5,3 / 5,4 / 5)
$$


and trace field $\mathbb{Q}$. We see from row \#35 of Table 5 that the obstruction vanishes and hence it is definable over $\mathbb{Q}$. We find the following concrete realization

$$
T_{0}=\left(\begin{array}{rrrr}
1 & 0 & 1 & 0 \\
1 & 1 & 1 & 1 \\
-1 & 0 & 0 & -1 \\
0 & 0 & 0 & 1
\end{array}\right), \quad T_{1}=\left(\begin{array}{rrrr}
1 & 0 & 1 & 0 \\
0 & 1 & 0 & 1 \\
-1 & 0 & 0 & 0 \\
0 & -1 & 0 & 0
\end{array}\right), \quad T_{\infty}=\left(\begin{array}{rrrr}
-1 & 0 & -1 & -1 \\
0 & 0 & 0 & -1 \\
1 & 0 & 0 & 0 \\
-1 & 1 & 0 & 0
\end{array}\right) .
$$

Computing the invariant Hermitian form, we find that these matrices are symplectic. Let $\Gamma:=\left\langle T_{0}, T_{1}, T_{\infty}\right\rangle \subseteq \mathrm{Sp}_{4}(\mathbb{Z})$ be the monodromy group.

We will show that $\mathcal{G}$ arises from $H^{1}$ of a family of genus two curves (so it is motivic). To find these, we use an argument we learned from D. Roberts. We will see that the group $\Gamma$ equals the monodromy of a finite monodromy G-II modulo 2 (denoted $\mathcal{G}_{1}$ below) and use it to produce a family of polynomials of degree 6 which give rise to the desired curves.

Bender [1] has given the following generators for the symplectic group $\mathrm{Sp}_{4}(\mathbb{Z})$.

$$
K:=\left(\begin{array}{rrrr}
1 & 0 & 0 & 0 \\
1 & -1 & 0 & 0 \\
0 & 0 & 1 & 1 \\
0 & 0 & 0 & -1
\end{array}\right), \quad L:=\left(\begin{array}{rrrr}
0 & 0 & -1 & 0 \\
0 & 0 & 0 & -1 \\
1 & 0 & 1 & 0 \\
0 & 1 & 0 & 0
\end{array}\right)
$$

In terms of these generators, we have

$$
T_{1}=\left(K L^{-2}\right)^{3} L^{-1}, \quad T_{\infty}=\left(K L^{-5}\right)^{4} .
$$

We can easily verify using MAGMA that the monodromy group $\Gamma:=\left\langle T_{0}, T_{1}, T_{\infty}\right\rangle \leq$ $\mathrm{Sp}_{4}(\mathbb{Z})$ is the unique subgroup of index two, namely the commutator subgroup of $\mathrm{Sp}_{4}(\mathbb{Z})$. Here are the calculations

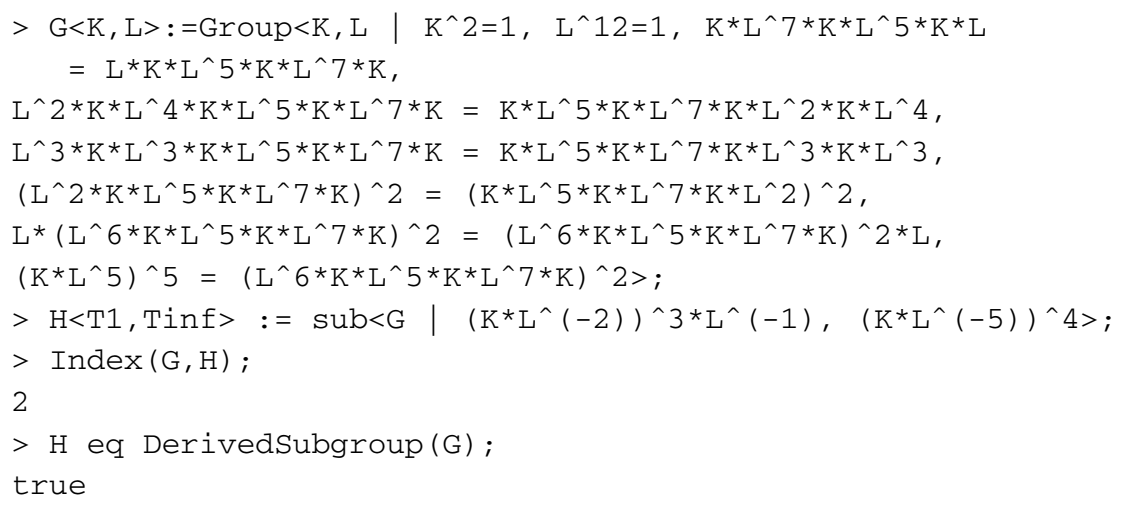

The quotient of $\mathrm{Sp}_{4}(\mathbb{Z})$ by its level two congruence subgroup is isomorphic to $\mathrm{Sp}_{4}\left(\mathbb{F}_{2}\right)$, which is known to be isomorphic to $S_{6}$. We see that $\Gamma$ maps surjectively to $A_{6}$ under the projection $\operatorname{map} f$.

$>\mathrm{U}:=$ SymmetricGroup (6);

$>$ homs $:=\operatorname{Homomorphisms}(\mathrm{G}, \mathrm{U}:$ Limit $:=1)$;

$>$ homs;

[

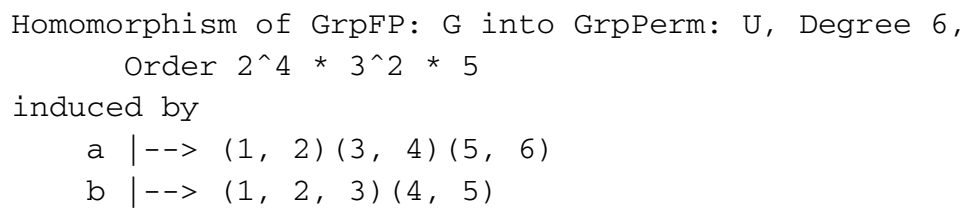


]

$>\mathrm{f}:=$ homs $[1]$;

$>\mathrm{f}(\mathrm{T} 1)$;

$(1,3,2)(4,5,6)$

$>\mathrm{f}(\mathrm{Tinf})$;

$(1,4,6,5,3)$

$>\mathrm{f}\left(\operatorname{Tinf} \wedge(-1) * \mathrm{~T}^{\wedge}(-1)\right)$;

$(2,3,4)$

Consider now the G-II rigid local system $\mathcal{G}_{1}$ with parameters

$$
\left(\alpha_{1}, \alpha_{2}\right)=(1 / 3,2 / 3), \quad\left(\beta_{1}, \beta_{2}\right)=(1 / 3,2 / 3), \quad\left(\gamma_{1}, \gamma_{2}, \gamma_{3}, \gamma_{4}\right)=(1 / 5,2 / 5,3 / 5,4 / 5)
$$

Its trace field is $\mathbb{Q}$, and indeed we find it in row \#5 of Table 6 among those with finite monodromy. Since $\mu=-1$, the system is not definable over $\mathbb{Q}$. Using a realization over $\mathbb{Q}(\sqrt{-3})$ with MAGMA, we find that the monodromy group is isomorphic to $\mathrm{SL}_{2}\left(\mathbb{F}_{9}\right)$, a central extension of $A_{6}$ by $C_{2}$. This group has two irreducible representations of degree four with Schur index two.

Here is the calculation with MAGMA.

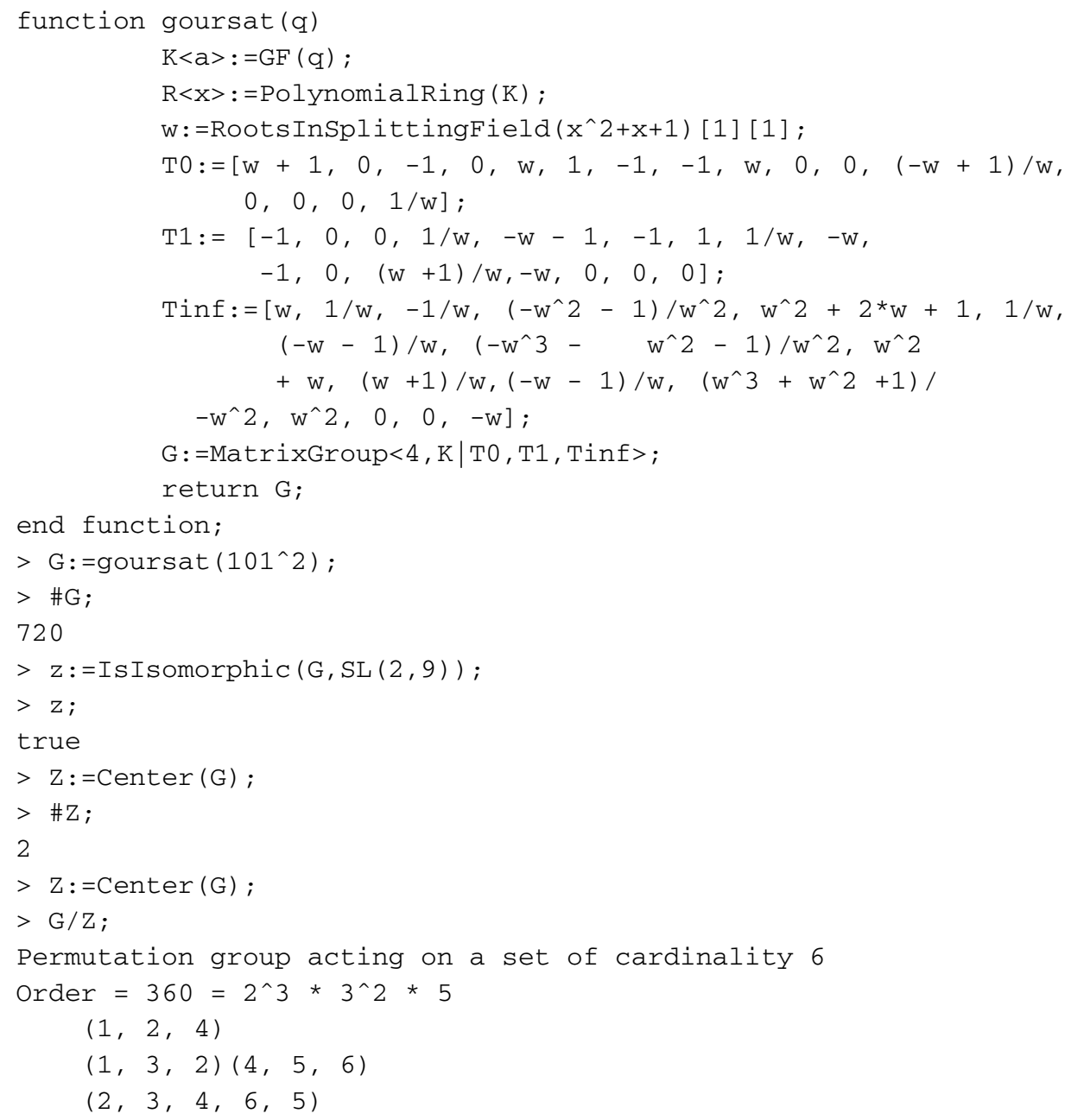

Note that the parameters for $\mathcal{G}$ and $\mathcal{G}_{1}$ are equal up to fractions with denominator 2. This means that their respective local monodromies are the same modulo 2 . It is clear, 
for example, that the monodromy of $\mathcal{G}_{1}$ is isomorphic to $A_{6} \cong \operatorname{PSL}_{2}\left(\mathbb{F}_{9}\right)$ modulo 2 as the center acts by \pm 1 .

The three even permutations

$$
\sigma_{0}:=f\left(T_{0}\right)=(2,3,4), \sigma_{1}:=f\left(T_{1}\right)=(1,3,2)(4,5,6), \sigma_{\infty}:=f\left(T_{\infty}\right)=(1,4,6,5,3)
$$

we computed above generate $A_{6}$ and correspond to a Belyi map with cycle type $31^{3}, 3^{2}, 51$. D. Roberts showed us how this map is given by the following polynomial

$$
P(x, t):=x^{3}\left(x^{3}+3 x^{2}-5\right)-t(3 x-1) .
$$

Indeed we have

$$
\begin{aligned}
P(x, 0) & =x^{3}\left(x^{3}+3 x^{2}-5\right), \\
P(x, 1) & =\left(x^{2}+x-1\right)^{3}, \\
P(x, \infty) & =3 x-1 .
\end{aligned}
$$

We consider the family of genus two curves defined by the hyperelliptic equation

$$
C_{t}: \quad y^{2}=4 P(x, t) .
$$

Its Igusa invariants are

$$
\begin{aligned}
J_{2} & =2^{2} \cdot 3 \cdot 5^{2} \cdot(4 t+1), \\
J_{4} & =2 \cdot 3 \cdot 5^{4} \cdot(4 t+1)^{2} \\
J_{6} & =2^{2} \cdot 5^{4} \cdot\left(736 t^{3}+2928 t^{2}-564 t+25\right), \\
J_{8} & =3 \cdot 5^{6} \cdot(4 t+1)\left(1856 t^{3}-8112 t^{2}+3156 t-25\right), \\
J_{10} & =2^{8} \cdot 3^{6} \cdot 5^{5} \cdot(t-1)^{4} t^{2} .
\end{aligned}
$$

By construction, the Galois representation on the two torsion of the Jacobian of $C_{t}$ for a generic $t \in \mathbb{Q}$ is congruent modulo two to that of the Artin representation associated to the Belyi map. We therefore expect that the motive $H^{1}\left(C_{t}, \mathbb{Q}\right)$ corresponds to $\mathcal{G}$.

We check that this indeed the case by computing the linear differential equation satisfied by periods of $C_{t}$. Starting with $\omega:=d x / y$ we apply $D:=d / d t$. reducing at each stage to a representative differential form of the type $p(x) / y d x$ with $p$ of degree at most five modulo exact differentials. We then look for a linear relation among $\omega, D \omega \ldots, D^{4} \omega$.

In this way, we find that $\omega$ is annihilated modulo exact differentials by the differential operator

$$
\begin{aligned}
& t^{2}(t-1)^{4} D^{4}+t(t-1)^{3}(9 t-5) D^{3}+\frac{1}{180}(t-1)^{2}\left(3456 t^{2}-3281 t+715\right) D^{2} \\
& +\frac{1}{450}(t-1)^{2}(3888 t-2473) D+\frac{1}{810000}\left(186624 t^{2}-378373 t+169874\right) .
\end{aligned}
$$

It is easy to verify that the differential equation is of the expected type G-II with exponents

$$
(-1 / 6,0,1 / 6,1), \quad(-1 / 6,1 / 6,5 / 6,7 / 6), \quad(2 / 5,3 / 5,4 / 5,6 / 5)
$$

at $t=0,1, \infty$, respectively. 
Table 8 Infinite families

\begin{tabular}{lllllll}
\hline & $\beta, \alpha_{1}, \alpha_{2}$ & $\gamma$ & $\Gamma / A$ & $A$ & $|Z(\Gamma)|$ & $\mid \mathrm{mpr}$ \\
\hline 1 & $1 / 2,1 / 2, r$ & $-r / 4,1 / 4-r / 4,1 / 2-r / 4,3 / 4-r / 4$ & $D_{4}$ & $\Delta_{1, n}$ & $n$ & $*$ \\
2 & $1 / 2,1 / 3,2 / 3$ & $r,-r / 3,1 / 3-r / 3,2 / 3-r / 3$ & $A_{4}$ & $\Delta_{2, n}$ & $\operatorname{gcd}(n, 4)$ & $*$ \\
\hline
\end{tabular}

\section{Infinite families}

Considering the stringent conditions required for the invariant Hermitian form $H$ to be definite, it can seem unlikely that there would be infinitely many examples where $H$ and all its Galois conjugates are definite. However, just as for hypergeometrics [2, Theorem 5.8], this is indeed the case. Moreover, again like hypergeometrics, they come in families all of which have the (finite) monodromy group $\Gamma \subseteq \mathrm{SL}_{4}(\mathbb{C})$ acting imprimitively (see Table 8 ). This is not surprising in light of Jordan's theorem (see the discussion at the end of Sect. 5.1).

In this table, $r=m / n$ is an arbitrary rational number in the lowest terms and

$$
\Delta_{1, n} \equiv\left\{\begin{array} { l l } 
{ C _ { n } ^ { 4 } } & { v _ { 2 } ( n ) = 0 , } \\
{ C _ { n / 2 } ^ { 3 } C _ { n } } & { v _ { 2 } ( n ) \geq 1 , }
\end{array} \quad \Delta _ { 2 , n } \equiv \left\{\begin{array}{ll}
C_{n}^{3}, & v_{2}(n)=0, \\
C_{n / 2}^{3} C_{2}, & v_{2}(n)=1, \\
C_{n / 4}^{3} C_{4}, & v_{2}(n) \geq 2,
\end{array}\right.\right.
$$

where $v_{2}$ is the valuation at 2 .

We will show that in fact there are such examples for all of the cases considered by Simpson [24] with $g=0, k=3$ punctures and one partition equal to $1^{n}$ for some $n$. For all of these systems, there are infinite families of examples all lying in a single geodesic in the positive components $\mathbb{T}_{+}^{\text {irr }}$.

\subsection{Rational powers}

We start by showing that the rational powers of algebraic functions satisfy differential equations of certain fixed order.

Proposition 4 Let $f(t)$ be an algebraic function of degree $m$. Then for all $r \in \mathbb{Q}$ the function $f^{r}$ satisfies $\mathcal{L}_{r} f^{r}=0$, where $\mathcal{L}_{r}$ is a differential operator of order $m$, whose coefficients depend polynomially on $r$.

Proof Let $P(t, y)=0$ be the defining equation for $f$, and let $y_{1}(t), \ldots, y_{m}(t)$ be its solutions. Denote by $W\left(f_{1}, \ldots, f_{n}\right)$ the Wronskian determinant and let us write

$$
\mathcal{L}_{r}[y]=\frac{W\left(y, y_{1}^{r}, \ldots, y_{m}^{r}\right)}{W\left(y_{1}^{r}, \ldots, y_{m}^{r}\right)}=y^{(m)}+A_{m-1} y^{(m-1)}+\cdots+A_{0} .
$$

Define polynomial differential operators $D_{n}$ by

$$
\frac{f_{i}^{(n)}}{f_{i}}=D_{n}\left(\frac{f_{i}^{\prime}}{f_{i}}\right) \text {. }
$$

Then $D_{0}(f)=1, D_{1}(f)=f, D_{2}(f)=f^{\prime}+f^{2}$, and in general they are defined by the recursion $D_{n+1}(f)=\left(D_{n}(f)\right)^{\prime}+D_{n}(f) f$. Using these operators we can write the Wronskian in terms of logarithmic derivatives as $W\left(f_{1}, \ldots, f_{n}\right)=f_{1} \ldots f_{n} \operatorname{det}\left(D_{i}\left(f_{j}^{\prime} / f_{j}\right)\right)_{i, j}$. Expanding the determinants in the definition of $\mathcal{L}_{r}$ shows that $A_{k}$ can be expressed as rational functions in $r$ whose coefficients are symmetric expressions in $y_{1}, \ldots, y_{m}$ and their derivatives and thus are rational functions of $t$.

For generic $r$ (more precisely, whenever $W\left(y_{1}^{r}, \ldots, y_{m}^{r}\right) \neq 0$ ) the functions $y_{1}^{r}, \ldots, y_{m}^{r}$ form the full space of solutions of $\mathcal{L}_{r}$, and thus, the singularities of $\mathcal{L}_{r}$ are contained in the 


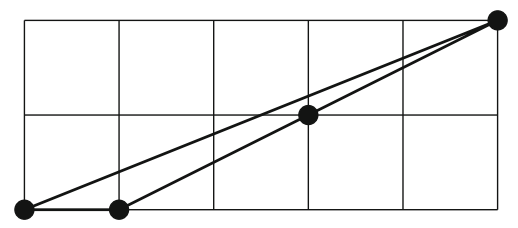

Fig. $1 \quad \Delta_{5}$

set of singularities of $y_{1}^{r}, \ldots, y_{m}^{r}$ together with the set of points $t$ where one of $y_{i}$ becomes 0 . In terms of the defining equation $P(t, y)=p_{m}(t) y^{m}+\cdots+p_{0}(t)$, these are exactly the values of $t$ where $p_{0}(t) p_{m}(t)$ or the discriminant of $P$ vanishes.

For instance, consider $u_{2}(\tau):=(\eta(2 \tau) / \eta(\tau))^{24}$. It is known that $u_{2}$ is a Hauptmodul for $\Gamma_{0}(2)$ and satisfies the algebraic equation

$$
A_{2}(u, t):=t(1+256 u)^{3}-1728 u=0, \quad t:=1728 / j,
$$

where $j$ is the standard elliptic $j$-invariant. For any $r \in \mathbb{Q}$, its $r$-th power is annihilated by the third-order differential operator

$$
x^{3}(x-1) \frac{d^{3}}{d x^{3}}+x^{2}(4 x-5 / 2) \frac{d^{2}}{d x^{2}}+x\left(\frac{20}{9} x+\frac{(3 r+2)(r-1)}{4}\right) \frac{d}{d x}+\frac{r^{2}(r-1)}{4},
$$

when expressed in $x=t(\tau)=1728 / j(\tau)$. The local exponents are

$$
0:(r,-r / 2,(1-r) / 2), \quad 1:(0,1 / 2,1), \quad \infty:(0,1 / 3,2 / 3) .
$$

Hence these are hypergeometric equations.

\subsection{Simpson even and odd families}

Given an odd positive integer $N>1$ consider the hypergeometric series

$$
f_{N}(t):={ }_{2} F_{1}\left(\begin{array}{cc}
\frac{N-1}{2 N} & \frac{N+1}{2 N} \\
\frac{3}{2} & 1
\end{array}\right) .
$$

It is an algebraic function of $t$ satisfying $P_{N}\left(f_{N}(t), t\right)=0$ for a polynomial $P_{N}(u, t) \in \mathbb{Q}[u, t]$. This polynomial $P_{N}$ can be given explicitly; the information we need is the shape of its Newton polygon $\Delta_{N}$, convex hull of the $(r, s) \in \mathbb{Z}^{2}$ for which the monomial $u^{r} t^{s}$ in $P_{N}$ has a nonzero coefficient. The polygon $\Delta_{N}$ is in fact the triangle of vertices $(0,0),(1,0),(N,(N-$ $1) / 2$ ). If we orient the boundary of the triangle counterclockwise starting at the origin the three sides have slopes $0,1 / 2,-\kappa_{N}$, where $\kappa_{N}:=(N-1) / 2 N$, respectively.

For example, for $N=5$ we find

$$
P_{5}(u, t)=16 u^{5} t^{2}-500 u^{3} t+3125 u-3125
$$

and its Newton polygon is $\Delta_{5}$ with sides of slope $0,1 / 2,-2 / 5$ (see Fig. 1).

At a zero or pole of $f_{N}(t)$ we have $t=0,1$ or $t=\infty$. Hence by Proposition $4, f_{N}(t)^{r}$ for $r \in \mathbb{Q}$ satisfies a linear differential equation of order $N$ with singularities only at $t=0,1, \infty$.

In general, the exponents at $t=0, \infty$ of the differential equation satisfied by an algebraic function $f$ of this kind can be read-off from its Newton polygon $\Delta$. It can be proved that these are as follows. 
Assume that the Newton polygon of $f$ has no vertical segments. Then there exist unique leftmost and rightmost vertices of $\Delta$, say $p, q$, respectively. Let $l$ be the line joining $p$ and $q$. We can distinguish the top and bottom sides of $\Delta$ as those above and below $l$, respectively.

For each slope $\kappa \in \mathbb{Q}$ of a side $\delta$ of the Newton polygon consider the sequence

$$
[\kappa]:=0-\kappa r, \frac{1}{d}-\kappa r, \ldots, \frac{e-1}{d}-\kappa r,
$$

where $d$ is the denominator of $\kappa$ and $e$ is the horizontal width of $\delta$.

The exponents at $t=0$ for $f^{r}$ are $\left[\kappa_{1}\right],\left[\kappa_{2}\right], \ldots$, where $\kappa_{1}, \kappa_{2}, \ldots$ runs over the slopes of the bottom sides. The exponents at $t=\infty$ are similarly determined by the slopes of the top sides. The exponents at $t=1$ are independent of $r$ and can be computed directly from the Newton polygon of $p(u, t+1)$.

In the case of $f_{N}$ the bottom slopes are $0,1 / 2$, the only top slope is $-\kappa_{N}$ and we obtain the following

$$
\begin{array}{r|l}
t & \text { exponents } \\
\hline 0 & 0,-r / 2,1 / 2-r / 2, \ldots,(N-2) / 2-r / 2 \\
1 & 0,1 / 2,1, \ldots,(N-1) / 2 \\
\infty & \kappa_{N} r, 1 / N+\kappa_{N} r, \ldots,(N-1) / N+\kappa_{N} r
\end{array} .
$$

For example, when $N=5$ these exponents are

$$
\begin{array}{r|l}
t & \text { exponents } \\
\hline 0 & 0,-r / 2,1 / 2-r / 2,1-r / 2,3 / 2-r / 2 \\
1 & 0,1 / 2,1,3 / 2,2 \\
\infty & 2 / 5 r, 1 / 5+2 / 5 r, 3 / 5+2 / 5 r, 3 / 5+2 / 5 r, 4 / 5+2 / 5 r
\end{array} .
$$

If $r \in \mathbb{Q}$ is not an integer then the multiplicity of these exponents is $(m, m, 1),(m+$ $1, m),(1, \ldots, 1)$, where $m:=(N-1) / 2$. These are precisely the multiplicities of Simpson's odd rank case family of rigid local systems. Hence we have obtained a geodesic completely contained in the positive components $\mathbb{T}_{+}^{\mathrm{irr}}$ of this system's parameter space.

A completely analogous discussion holds for $N$ even with the same definition of $f_{N}$. Here it is more convenient to consider the algebraic equation for $f_{N}^{2}$, which is the hypergeometric function

$$
f_{N}(t)^{2}={ }_{2} F_{1}\left(\begin{array}{cc}
\frac{N-1}{N} & \frac{N+1}{N} \\
\frac{3}{2} & 2
\end{array}\right)
$$

It satisfies an algebraic equation of degree $N$ with Newton polygon the triangle of vertices $(0,0),(1,0),(N, N)$. The exponents are the same as in the case $N$ odd. The multiplicities however are now $(1, m-1, m),(m, m),(1, \ldots, 1)$, where $m:=N / 2$. These are the multiplicities of Simpson's even rank case family of rigid local systems [24]. Again we have obtained a geodesic completely contained in the positive components $\mathbb{T}_{+}^{\text {irr }}$ of the parameter space. For example, for $N=4$ we get a geodesic for Goursat G-II up to a twist.

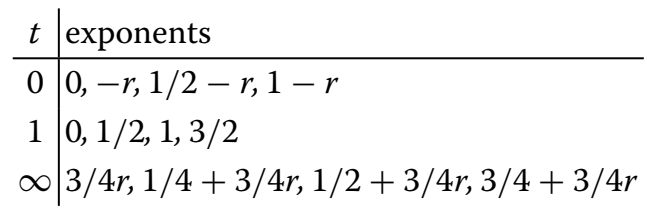

\subsection{Simpson extra case of rank 6}

The Hauptmodul $u_{5}:=(\eta(5 \tau) / \eta(\tau))^{6}$ satisfies the equation

$$
A_{5}(u, t)=t\left(3125 u^{2}+250 u+1\right)^{3}-1728 u=0,
$$


whose Newton polygon is a triangle with vertices $(0,1),(4,1),(0,1)$ and slopes $-1,1 / 5,0$.

Fractional powers of $u_{5}(\tau)=(\eta(5 \tau) / \eta(\tau))^{6}$ give the rank 6 extra case rigid local system of Simpson. Explicitly, in terms of $x=1 / t(\tau), u_{5}^{r}$ satisfies the differential equation

$$
\begin{aligned}
x^{4}( & -1)^{2} \frac{d^{6}}{d x^{6}}+x^{3}(x-1)(17 x-10) \frac{d^{5}}{d x^{5}} \\
+ & x^{2}\left(\frac{-3 r^{2}+2 r+432}{5} x^{2}+\frac{108 r^{2}-72 r-18377}{180} x+\frac{220}{9}\right) \frac{d^{4}}{d x^{4}} \\
+ & x\left(\frac{8 r^{3}-118 r^{2}+74 r+3720}{25} x^{2}\right. \\
& \left.-\frac{576 r^{3}-5796 r^{2}+3528 r+209915}{1800} x+\frac{40}{3}\right) \frac{d^{3}}{d x^{3}} \\
+ & \left(\frac{-45 r^{4}+860 r^{3}-5145 r^{2}+2950 r+45024}{625} x^{2}\right. \\
& \left.-\frac{576 r^{3}-2676 r^{2}+1448 r+26135}{900} x+\frac{40}{81}\right) \frac{d^{2}}{d x^{2}} \\
+ & \left(\frac{24 r^{5}-415 r^{4}+2790 r^{3}-7925 r^{2}+3846 r+15120}{3125} x\right. \\
& \left.-\frac{64 r^{3}-164 r^{2}+72 r+315}{900}\right) \frac{d}{d x} \\
& -\frac{r^{2}(r-1)(r-2)(r-3)(r-4)}{3125} .
\end{aligned}
$$

The exponents of this equation for generic $r \in \mathbb{Q}$ are

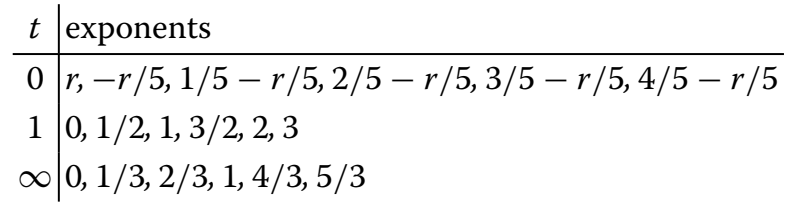

with multiplicities $(4,2),(2,2,2),(1, \ldots, 1)$. This is then a geodesic in the positive components $\mathbb{T}_{+}^{\text {irr }}$ of Simpson's extra case. A special case reduces to a hypergeometric series

$$
\frac{u_{5}}{t}={ }_{3} F_{2}\left(\begin{array}{ccc}
\frac{1}{2} & \frac{1}{6} & \frac{5}{6} \\
\frac{4}{5} & \frac{6}{5} & 1
\end{array} \mid t\right)^{6} .
$$

For $r=1,3$, the equation reduces to a hypergeometric equation of order 3, and for $r=2,4,8,14$ it reduces to an equation of order 5 with rigid monodromy of Simpson's odd type.

\subsection{Hypergeometric}

Theorem 5.8 in [2] describes a geodesic in the case of hypergeometric rigid local systems. This can be made explicit in terms of fractional powers of a fixed algebraic function like all the previous examples. We have already encountered one case (see the example at the end of Sect. 12.1). We illustrate this further with an instance of rank 5.

Consider the algebraic equation

$$
u(1-u)^{4}-\frac{4^{4}}{5^{5}} t=0 .
$$


We can solve for $u$ as a function of $t$ by inversion. Let

$$
f(t)=\frac{5^{5}}{4^{4}} \frac{u(t)}{t}=1+t_{1}+\frac{13}{8} t_{1}^{2}+\frac{51}{16} t_{1}^{3}+\frac{1771}{256} t_{1}^{4}+\frac{4095}{256} t_{1}^{5}+O\left(t_{1}^{6}\right), \quad t_{1}:=\frac{5^{5}}{4^{4}} t .
$$

We find that

$$
f(t)={ }_{4} F_{3}\left(\begin{array}{cccc}
\frac{4}{5} & \frac{6}{5} & \frac{7}{5} & \frac{8}{5} \\
\frac{3}{2} & \frac{5}{4} & \frac{7}{4} & 2
\end{array}\right)
$$

We compute the local exponents of $f^{r}$ for $r \in \mathbb{Q}$ and find

$$
\begin{array}{r|l}
t & \text { exponents } \\
\hline 0 & 0,-r, 1 / 4-r, 1 / 2-r, 3 / 4-r \\
1 & 1 / 2,0,1,2,3 \\
\infty & 4 / 5 r, 1 / 5+4 / 5 r, 2 / 5+4 / 5 r, 3 / 5+4 / 5 r, 4 / 5+4 / 5 r
\end{array}
$$

We obtain the following identity between hypergeometric functions

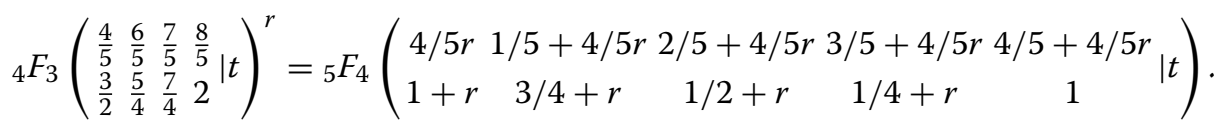

\subsection{Goursat II}

There is another geodesic in the case G-II apart from that in Sect. 12.2 for $n=4$. Consider the modular unit $u_{3}(\tau):=(\eta(3 \tau) / \eta(\tau))^{12}$. It is a classical fact that $u_{3}$ is a Hauptmodul for the modular curve $X_{0}(3)$ and satisfies the algebraic equation $A_{3}\left(3^{6} u_{3}, 1728 j^{-1}\right)=0$, where

$$
A_{3}(u, t):=t(u+27)(u+3)^{3}-1728 u .
$$

The Newton polygon of $A_{3}$ is a triangle with vertices $(0,1),(4,1),(0,1)$ and slopes $-1,1 / 3,0$. The fourth-order differential equation satisfied by $u_{3}^{r}$ is

$$
\begin{aligned}
& x^{2}(x-1)^{2} \frac{d^{4}}{d x^{4}}+x(x-1)(7 x-4) \frac{d^{3}}{d x^{3}} \\
& +\left(\frac{-6 r^{2}+3 r+92}{9} x^{2}+\frac{24 r^{2}-12 r-421}{36} x+\frac{20}{9}\right) \frac{d^{2}}{d x^{2}} \\
& +\left(\frac{8 r^{3}-33 r^{2}+13 r+60}{27} x-\frac{64 r^{3}-192 r^{2}+68 r+345}{216}\right) \frac{d}{d x} \\
& \quad-\frac{r^{2}(r-1)(r-2)}{27},
\end{aligned}
$$

when expressed in $x=1 / t(\tau)=j(\tau) / 1728$. The exponents of this equation for generic $r \in \mathbb{Q}$ are

$$
\begin{array}{r|l}
t & \text { exponents } \\
\hline 0 & 0,1 / 3,2 / 3,1 \\
1 & 0,1 / 2,1,3 / 2 \\
\infty & r,-r / 3,1 / 3-r / 3,2 / 3-r / 3
\end{array}
$$

We have

$$
\frac{u_{3}}{t}={ }_{2} F_{1}\left(\begin{array}{cc}
\frac{7}{12} & \frac{1}{4} \\
\frac{4}{3} & 1
\end{array} \mid t\right), \quad t:=\frac{1728}{j} .
$$

Here is a detailed description of this geodesic. The four exponents at $\infty$ are

$$
\gamma_{1}=3 r, \quad \gamma_{2}=-r, \quad \gamma_{3}=-r+1 / 3, \quad \gamma_{4}=-r+2 / 3 .
$$




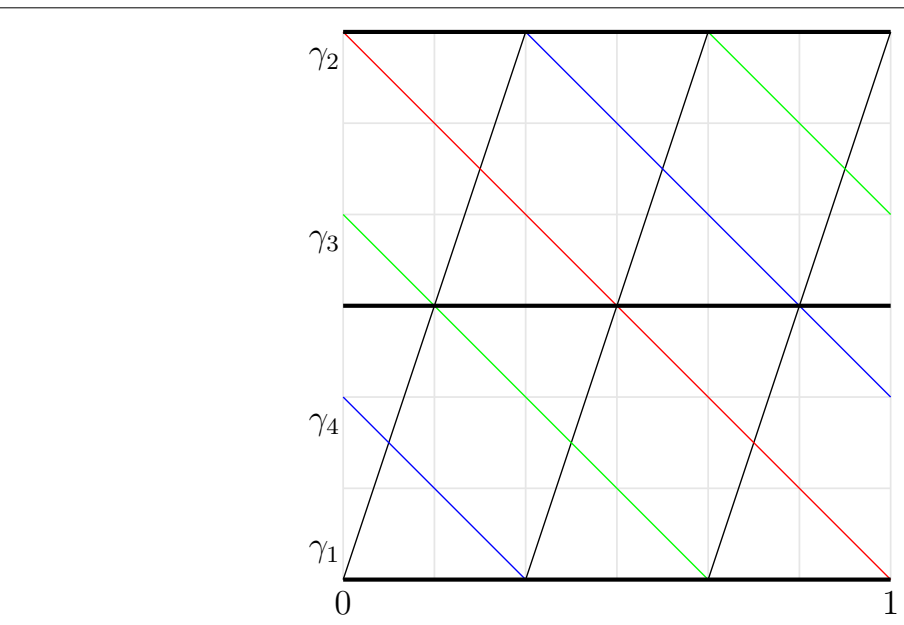

Fig. 2 G-ll geodesic $\gamma$

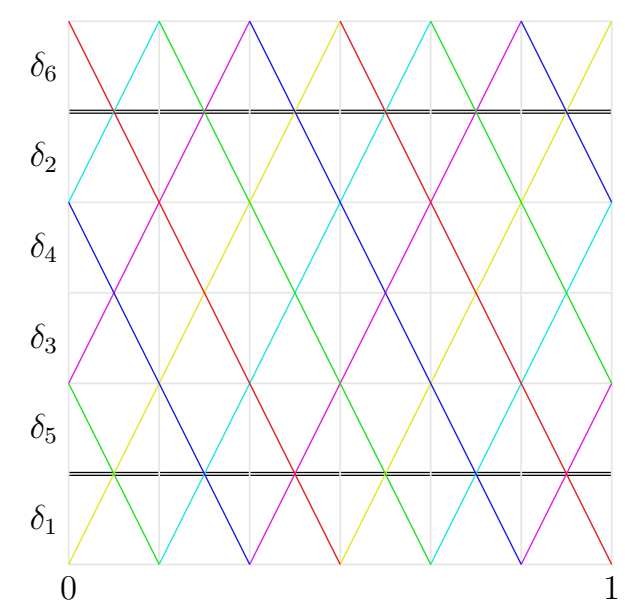

Fig. 3 G-ll geodesic $\delta$

We plot these modulo $\mathbb{Z}$ as a function of $r$ (see Fig. 2). The first condition $\left(n_{1}=2\right.$ in the notation of (3)) for these parameters for generic $r$ to be in the positive components $\mathbb{T}_{+}^{\mathrm{irr}}$ is that there are two in each of the indicated horizontal strips. This is visible in the plot.

The second condition for positive definiteness involves the parameters

$$
\begin{aligned}
\delta_{1}=2 r, \quad \delta_{2}=2 r+2 / 3, \quad \delta_{3}=2 r+1 / 3, \\
\quad \delta_{4}=-2 r+2 / 3, \quad \delta_{5}=-2 r+1 / 3, \quad \delta_{6}=-2 r .
\end{aligned}
$$

For generic $r$ there should be four $\delta$ 's in the interval $(1 / 6,5 / 6)$. A plot of these as functions of $r$ modulo $\mathbb{Z}$ is given in Fig. 3 where this condition is visible.

\section{Author details}

${ }^{1}$ Max Planck Institute for Mathematics, Vivatsgasse 7, 53111 Bonn, Germany, ${ }^{2}$ The Abdus Salam International Centre for Theoretical Physics, Strada Costiera 11, 34151 Trieste, Italy.

\section{Acknowledgements}

Open access funding provided by Max Planck Society. This work was started at the Abdus Salam Centre for Theoretical Physics and completed during the special trimester Periods in Number Theory, Algebraic Geometry and Physics at the Hausdorff Institute of Mathematics in Bonn, Germany. We would like to thank these institutions as well as the Max Planck 
Institute for Mathematics in Bonn for their hospitality and support. We thank the anonymous referee for helpful comments. The second author would like to thank N. Katz and D. Roberts for useful exchanges regarding the subject of this work.

\section{Received: 6 March 2018 Accepted: 17 August 2018 Published online: 5 September 2018}

\section{References}

1. Bender, P.: Eine Präsentation der symplektischen Gruppe $\mathrm{Sp}(4, Z)$ mit 2 Erzeugenden und 8 definierenden Relationen. J. Algebra 65, 328-331 (1980)

2. Beukers, F., Heckman, G.: Monodromy for the hypergeometric function ${ }_{n} F_{n-1}$. Invent. Math. 95, 325-354 (1989)

3. Bosma, W., Cannon, J., Playoust, C.: The Magma algebra system I: the user language. J. Symb. Comput. 24(3-4), 235-265 (1997)

4. Boulanger, A.: Contribution à l'étude des équations différentielles linéaires et homogènes intégrables algebriquement. Gauthier-Villars, Paris (1897)

5. Crawley-Boevey, W.: Indecomposable parabolic bundles and the existence of matrices in prescribed conjugacy class closures with product equal to the identity. Publ. Math. Inst. Hautes tudes Sci. 100, 171-207 (2004)

6. Deligne, P.: Équations différentielles à points singuliers réguliers. Lecture Notes in Mathematics, vol. 163. Springer, Berlin-New York (1970)

7. Elkies, N.: The Klein quartic in number theory. The eightfold way, pp. 51-101, Math. Sci. Res. Inst. Publ., vol. 35. Cambridge Univ. Press, Cambridge (1999)

8. Esnault, H., Groechenig, M.: Cohomologically rigid local systems and integrality, arXiv:1711.06436v3

9. Gleizer, O.: Some explicit solutions of the additive Deligne-Simpson problem and their applications. Adv. Math. 178 $311-374$ (2003)

10. Goursat, É.: Sur les fonctions d'une variable analogues aux fonctions hypergéométriques. Ann. Sci. IÉ.N.S. $3^{e}$ Sér. 3 107-136 (1886)

11. Hanany, A., He, Y.-H.: A monograph on the classification of the discrete subgroups of SU(4). J. High Energy Phys. 2, 12 (2001)

12. Haraoka, Y.: Canonical forms of differential equations free from accessory parameters SIAM. J. Math. Anal. 25, 12031226 (1994)

13. Haraoka, Y.: Monodromy representations of systems of differential equations free from accessory parameters. SIAM J. Math. Anal. 25, 1595-1621 (1994)

14. Hausel, T., Letellier, E., Rodriguez Villegas, F.: Arithmetic harmonic analysis on character and quiver varieties math.RT/0810.2076. Duke Math. J. 160, 323-400 (2011)

15. Hurwitz, A.: Über einige besondere homogene lineare Differentialgleichungen. Math. Ann. 26, 117-126 (1869)

16. Katz, N.M.: Rigid Local Systems, vol. 139. Annals of Mathematics Studies (1996)

17. Liouville, J.: Mémoire sur lintégration dune classe déquations différentielles du second ordre en quantités finies explicites. J. Math. Pures Appl. 4, 423-456 (1839)

18. Oshima, T.: Fractional Calculus of Weyl Algebra and Fuchsian Differential Equations. MSJ Memoirs, vol. 28. Mathematical Society of Japan, Tokyo (2012)

19. Peters, C.A.M., Steenbrink, J.H.M.: Monodromy of variations of Hodge structure. Acta Appl. Math. 75, 183-194 (2003)

20. Roberts, D.: Rigid Jordan tuples, Preprint http://cda.morris.umn.edu/ deroberts/

21. Schwarz, H.A.: Über diejenigen Fälle, in welchen die Gaussische hypergeometrische Reihe eine algebraische Function ihres vierten Elementes darstellt. J. Reine Angew. Math. 75, 292-335 (1873)

22. Serre, J.-P.: Three letters to Walter Feit on group representations and quaternions. J. Algebra 319(2), $549-557$ (2008)

23. Serre, J.-P.: Local Fields. Graduate Texts in Mathematics, vol. 67. Springer, New York-Berlin (1979)

24. Simpson, C.: Products of matrices. AMS Proc. 1, 157-185 (1992)

25. Simpson, C.: Higgs bundles and local systems. Publ. Math. IHES 75, 5-95 (1992)

26. Singer, M.F.: Algebraic solutions of nth order linear differential equations. In: Proceedings of the Queen's University 1979 Conference on Number Theory, Queens Papers in Pure and Applied Mathematics, vol. 54, pp. 379-420

27. The PARI Group, PARI/GP version 2.9.4, Univ. Bordeaux (2018). http://pari.math.u-bordeaux.fr/

28. Zagier, D.: Elliptic modular forms and their applications. In: Ranestad, K. (ed.) The 1-2-3 of Modular Forms, pp. 1-103. Universitext. Springer, Berlin (2008)

29. Zagier, D.: Arithmetic and topology of differential equations. In: Mehrmann, V., Skutella, M. (eds.) European Mathematical Society, pp. 717-776, Berlin. Proceedings of the Seventh European Congress of Mathematics (Berlin, July 18-22, 2016) (2018) 